\title{
Biomimetic Synthesis Enables the Structure Revision of Furoerioaustralasine
}

Matthew A. Coleman, Laura Burchill, Christopher J. Sumby and Jonathan H. George*

Department of Chemistry, University of Adelaide, Adelaide, SA 5005, Australia.

\section{Supporting Information}

\section{Table of Contents}

1. General methods 2

2. Experimental procedures 3

$\begin{array}{ll}3 . & \text { NMR spectra } \\ & 10\end{array}$

4. Comparison of natural and synthetic NMR data 35

5. Single crystal X-ray data 37 


\section{General methods}

All chemicals were purchased from commercial suppliers and used as received. All reactions were performed under an inert atmosphere of $\mathrm{N}_{2}$. All organic extracts were dried over anhydrous magnesium sulfate. Thin layer chromatography was performed using aluminium sheets coated with silica gel $F_{254}$. Visualization was aided by viewing under a UV lamp and staining with ceric ammonium molybdate or $\mathrm{KMnO}_{4}$ stain followed by heating. All $\mathrm{R}_{\mathrm{f}}$ values were measured to the nearest 0.05 . Flash column chromatography was performed using 40-63 micron grade silica gel. Melting points were recorded on a digital melting point apparatus and are uncorrected. Infrared spectra were recorded using an FT-IR spectrometer as the neat compounds. High field NMR spectra were recorded using either a $500 \mathrm{MHz}$ spectrometer $\left({ }^{1} \mathrm{H}\right.$ at $500 \mathrm{MHz},{ }^{13} \mathrm{C}$ at $125 \mathrm{MHz}$ ) or $600 \mathrm{MHz}$ spectrometer $\left({ }^{1} \mathrm{H}\right.$ at $600 \mathrm{MHz},{ }^{13} \mathrm{C}$ at $\left.150 \mathrm{MHz}\right)$. The solvent used for NMR spectra was $\mathrm{CDCl}_{3}$ unless otherwise specified. ${ }^{1} \mathrm{H}$ chemical shifts are reported in ppm on the $\delta$-scale relative to TMS $(\delta 0.0)$ or $\mathrm{CDCl}_{3}(\delta 7.26)$ and ${ }^{13} \mathrm{C} \mathrm{NMR}$ chemical shifts are reported in ppm relative to $\mathrm{CDCl}_{3}(\delta 77.16)$. Multiplicities are reported as (br) broad, (s) singlet, (d) doublet, (t) triplet, (q) quartet, (quin) quintet, (sext) sextet, (hept) heptet and (m) multiplet. All $J$-values were rounded to the nearest $0.1 \mathrm{~Hz}$. ESI high resolution mass spectra were recorded on an ESI-TOF mass spectrometer. 


\section{Experimental procedures}

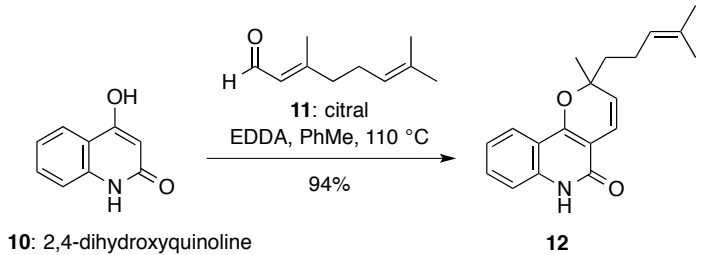

To a solution of 2,4-dihydroxyquinoline (10) (9.03 g, $56.1 \mathrm{mmol}, 1.0$ equiv.) in PhMe (300 mL) was added ethylenediamine diacetate (1.03 g, $5.72 \mathrm{mmol}, 0.10$ equiv.) and citral (11) $(9.60 \mathrm{~mL}, 56.1$ mmol, 1.0 equiv.). The resultant solution was stirred at reflux for $4 \mathrm{~h}$. The mixture was then cooled to room temperature and concentrated in vacuo. The residue was purified via flash column chromatography on $\mathrm{SiO}_{2}$ (3:2, hexanes:EtOAc) to afford $\mathbf{1 2}$ as an orange oil (15.5 g, $52.5 \mathrm{mmol}$, 94\%). Data for 12 matched that previously reported in the literature. ${ }^{1}$

\section{Data for 12:}

$\mathbf{R}_{\mathbf{f}}: 0.15$ (1:1, hexanes:EtOAc).

FTIR (neat): 2971, 1632, 1599, 1497, 1432, 1413, 1360, 1275, 1182, 1115, 1074, 908, 751, $729 \mathrm{~cm}^{-1}$. ${ }^{1}$ H NMR (500 MHz, CDCl $): \delta 11.57$ (br s, $\left.1 \mathrm{H}\right), 7.87$ (dd, $\left.J=8.1,1.4 \mathrm{~Hz}, 1 \mathrm{H}\right), 7.47$ (td, $J=7.2,1.2$ $\mathrm{Hz}, 1 \mathrm{H}), 7.34(\mathrm{~d}, J=7.9 \mathrm{~Hz}, 1 \mathrm{H}), 7.19$ (t, $J=7.9,1 \mathrm{H}), 6.81$ (d, $J=10.1 \mathrm{~Hz}, 1 \mathrm{H}), 5.50(\mathrm{~d}, J=10.1$ $\mathrm{Hz}, 1 \mathrm{H}), 5.10$ (tt, $J=7.1,1.3 \mathrm{~Hz}, 1 \mathrm{H}), 2.16$ (q, $J=7.9 \mathrm{~Hz}, 2 \mathrm{H}), 1.89-1.73(\mathrm{~m}, 2 \mathrm{H}), 1.63$ (s, 3H), $1.55(\mathrm{~s}, 3 \mathrm{H}), 1.51(\mathrm{~s}, 3 \mathrm{H}) \mathrm{ppm}$.

${ }^{13}$ C NMR (125 MHz, $\left.\mathbf{C D C l}_{3}\right): \delta 163.1,157.6,138.3,132.0,130.8,125.1,123.9,122.5,122.1,117.8$, $116.3,115.2,105.4,81.7,41.7,27.2,25.7,22.7,17.7$ ppm.

HRMS (ESI): calculated for $\mathrm{C}_{19} \mathrm{H}_{21} \mathrm{NO}_{2}[\mathrm{M}+\mathrm{H}]^{+} 296.1645$, found 296.1648 .

\footnotetext{
${ }^{1}$ Wang, X.; Lee, Y. R. Synthesis 2007, 3044.
} 


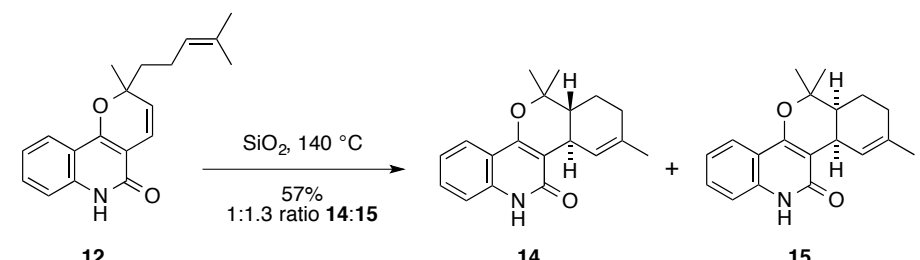

To a solution of 12 (14.2 g, 48.1 mmol, 1.0 equiv.) in $\mathrm{Et}_{2} \mathrm{O}(400 \mathrm{~mL})$ was added $\mathrm{SiO}_{2}(42.6 \mathrm{~g}, 300 \%$ $\mathrm{w} / \mathrm{w}$ ). The slurry was concentrated in vacuo, and then heated at $140{ }^{\circ} \mathrm{C}$ under $\mathrm{N}_{2}$ for $7 \mathrm{~h}$. After this time the mixture was directly loaded onto a column of $\mathrm{SiO}_{2}$ and purified via flash chromatography (4:1, $\mathrm{CH}_{2} \mathrm{Cl}_{2}$ :EtOAc) to give alkenes $\mathbf{1 4}$ and $\mathbf{1 5}$ as a 1:1.3 mixture of diastereomers $(8.13 \mathrm{~g}, 27.5$ mmol, 57\%). Repeated recrystallization from acetone afforded 15 as a white solid (4.56 g, $15.4 \mathrm{mmol}$, $32 \%$ ), whilst the filtrate containing $\mathbf{1 4}$ was recrystallized from $\mathrm{Et}_{2} \mathrm{O}$ to afford $\mathbf{1 4}$ as a white solid (3.20 g, $10.8 \mathrm{mmol}, 23 \%)$.

\section{Data for 14:}

$\mathbf{R}_{\mathbf{f}}: 0.50$ (1:2, hexanes:EtOAc).

FTIR (neat): 2888, 1634, 1602, 1497, 1430, 1385, 1271, 1120, 1090, 907, 839, 754, 728, $689 \mathrm{~cm}^{-1}$.

${ }^{1}$ H NMR (500 MHz, CDCl $): \delta 12.07$ (br s, $\left.1 \mathrm{H}\right), 7.88(\mathrm{~d}, J=8.0 \mathrm{~Hz}, 1 \mathrm{H}), 7.45$ (t, $\left.J=7.4 \mathrm{~Hz}, 1 \mathrm{H}\right)$, $7.42-7.38(\mathrm{~m}, 1 \mathrm{H}), 6.69$ (d, $J=3.8 \mathrm{~Hz}, 1 \mathrm{H}), 3.29$ (d, $J=10.8 \mathrm{~Hz}, 1 \mathrm{H}), 2.20(\mathrm{~m}, 2 \mathrm{H}), 1.95(\mathrm{~m}, 1 \mathrm{H})$, $1.72(\mathrm{~s}, 3 \mathrm{H}), 1.77-1.72(\mathrm{~m}, 1 \mathrm{H}), 1.57(\mathrm{~s}, 3 \mathrm{H}), 1.54-1.46(\mathrm{~m}, 1 \mathrm{H}), 1.20(\mathrm{~s}, 3 \mathrm{H}) \mathrm{ppm}$.

${ }^{13} \mathbf{C}$ NMR (150 MHz, $\left.\mathbf{C D C l}_{3}\right): \delta$ 157.6, 137.6, 133.7, 130.1, 129.4, 122.9, 122.7, 121.7, 116.2, 115.6, $107.7,80.3,45.6,33.6,31.5,27.4,24.9,23.5,20.0 \mathrm{ppm}$.

HRMS (ESI): calculated for $\mathrm{C}_{19} \mathrm{H}_{21} \mathrm{NO}_{2}[\mathrm{M}+\mathrm{H}]^{+} 296.1645$, found 296.1544 .

\section{Data for 15:}

$\mathbf{R}_{\mathbf{f}}: 0.50$ ( $1: 2$, hexanes:EtOAc).

FTIR (neat): 2888, 2853, 1635, 1604, 1497, 1430, 1387, 1367, 1269, 1212, 1171, 1131, $1099 \mathrm{~cm}^{-1}$.

${ }^{1}$ H NMR (600 MHz, CDCl $): \delta 11.63(\mathrm{~s}, 1 \mathrm{H}), 7.86(\mathrm{~d}, J=8.1,1 \mathrm{H}), 7.44(\mathrm{t}, J=7.3 \mathrm{~Hz}, 1 \mathrm{H}), 7.34(\mathrm{~d}$, $J=8.1 \mathrm{~Hz}, 1 \mathrm{H}), 7.15$ (t, $J=7.3 \mathrm{~Hz}, 1 \mathrm{H}), 6.60(\mathrm{~d}, J=5.2 \mathrm{~Hz}, 1 \mathrm{H}), 3.61(\mathrm{~m}, 1 \mathrm{H}), 2.09-1.86(\mathrm{~m}, 3 \mathrm{H})$, 1.83 (ddd, $J=11.9,6.1,2.9 \mathrm{~Hz}, 1 \mathrm{H}), 1.70$ (s, 3H), 1.54 (s, 3H), 1.36 (s, 4H) ppm.

${ }^{13}$ C NMR (150 MHz, $\left.\mathbf{C D C l}_{3}\right): \delta$ 165.2, 156.2, 137.5, 134.3, 129.9, 122.7, 121.6, 121.5, 116.1, 115.5, $108.9,78.9,40.0,31.4,29.9,26.0,25.2,23.8,20.6 \mathrm{ppm}$.

HRMS (ESI): calculated for $\mathrm{C}_{19} \mathrm{H}_{21} \mathrm{NO}_{2}[\mathrm{M}+\mathrm{H}]^{+} 296.1645$, found 296.1642 . 


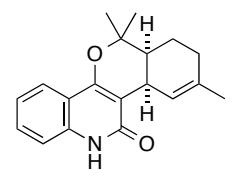

15

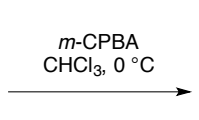

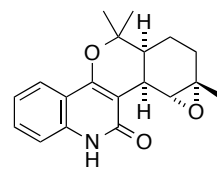

$16(47 \%)$

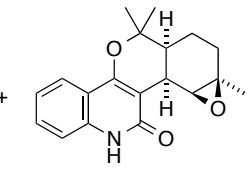

$17(18 \%)$

To a solution of 15 (132 mg, $0.447 \mathrm{mmol}, 1.0$ equiv.) in $\mathrm{CHCl}_{3}(20 \mathrm{~mL})$ was added $m$-CPBA (134 $\mathrm{mg}, 0.596 \mathrm{mmol}, 1.3$ equiv., $77 \%$ ) at $0{ }^{\circ} \mathrm{C}$ under $\mathrm{N}_{2}$. After $3 \mathrm{~h}$, the reaction was quenched with sat. $\mathrm{Na}_{2} \mathrm{SO}_{3(\text { aq })}(20 \mathrm{~mL})$, and the mixture was extracted with $\mathrm{CH}_{2} \mathrm{Cl}_{2}(2 \times 20 \mathrm{~mL})$. The combined organic extracts were washed with sat. $\mathrm{NaHCO}_{3(\mathrm{aq})}(50 \mathrm{~mL})$ and brine $(50 \mathrm{~mL})$, dried over anhydrous $\mathrm{MgSO}_{4}$, filtered and concentrated in vacuo to afford a crude mixture of $\mathbf{1 6}$ and $\mathbf{1 7}$ in a ratio of 2.2:1 (as determined by ${ }^{1} \mathrm{H}$ NMR). Purification via flash chromatography (EtOAc) then afforded $\mathbf{1 6}$ as a white solid (66.1 mg, $0.212 \mathrm{mmol}, 47 \%$ ) and $\mathbf{1 7}$ as a white solid (25.1 mg, $0.081 \mathrm{mmol}, 18 \%$ ).

\section{Data for 16:}

$\mathbf{R}_{\mathbf{f}}: 0.45$ (EtOAc).

FTIR (neat): 2974, 1638, 1608, 1499, 1433, 1396, 1274, 1195, 1174, 1116, 1086, 1054, $923 \mathrm{~cm}^{-1}$.

${ }^{1}$ H NMR (600 MHz, CDCl $)$ ): $\delta 12.38$ (br s, $\left.1 \mathrm{H}\right), 7.86(\mathrm{~d}, J=8.0 \mathrm{~Hz}, 1 \mathrm{H}), 7.47$ (td, $J=7.6,6.9,1.4$ $\mathrm{Hz}, 1 \mathrm{H}), 7.40$ (dd, $J=7.7,2.5 \mathrm{~Hz}, 1 \mathrm{H}), 7.17$ (t, $J=7.6 \mathrm{~Hz}, 1 \mathrm{H}), 4.49(\mathrm{~m}, 1 \mathrm{H}), 3.62(\mathrm{~d}, J=10.5 \mathrm{~Hz}$, 1H), $2.27-2.16(\mathrm{~m}, 2 \mathrm{H}), 1.94(\mathrm{ddd}, J=14.1,6.0,3.3 \mathrm{~Hz}, 1 \mathrm{H}), 1.76-1.73(\mathrm{~m}, 1 \mathrm{H}), 1.47$ (s, 3H), 1.38 (s, 3H), $1.55-1.46(\mathrm{~m}, 1 \mathrm{H}), 1.29(\mathrm{~s}, 3 \mathrm{H})$.

${ }^{13} \mathbf{C}$ NMR (150 MHz, $\left.\mathbf{C D C l}_{3}\right): \delta 165.7,158.4,137.8,130.6,122.7,121.9,115.8,115.7,104.8,79.3$, $61.3,58.6,36.2,32.2,27.6,25.8,25.6,24.2,18.9 \mathrm{ppm}$.

HRMS (ESI): calculated for $\mathrm{C}_{19} \mathrm{H}_{21} \mathrm{NO}_{3}[\mathrm{M}+\mathrm{H}]^{+} 312.1555$, found 312.1556 .

\section{Data for 17:}

R: 0.25 (EtOAc).

FTIR (neat): 2978, 1640, 11603, 1390, 1279, 1180, 1123, 1086, 976, 922, $834 \mathrm{~cm}^{-1}$.

${ }^{1} \mathbf{H}$ NMR (600 MHz, $\left.\mathbf{C D C l}_{3}\right): \delta 10.56(\mathrm{~s}, 1 \mathrm{H}), 7.81(\mathrm{dd}, J=8.1,1.4 \mathrm{~Hz}, 1 \mathrm{H}), 7.41(\mathrm{td}, J=7.2,1.2$ $\mathrm{Hz}, 1 \mathrm{H}), 7.22(\mathrm{dd}, J=8.2,1.1 \mathrm{~Hz}, 1 \mathrm{H}), 7.13(\mathrm{td}, J=7.2,1.2 \mathrm{~Hz}, 1 \mathrm{H}), 4.15(\mathrm{~d}, J=4.1 \mathrm{~Hz}, 1 \mathrm{H}), 3.58-$ $3.55(\mathrm{~m}, 1 \mathrm{H}), 2.04-1.99(\mathrm{~m}, 1 \mathrm{H}), 1.70-1.67(\mathrm{~m}, 1 \mathrm{H}), 1.57$ (d, $J=5.8 \mathrm{~Hz}, 2 \mathrm{H}), 1.50(\mathrm{~s}, 3 \mathrm{H}), 1.38(\mathrm{~s}$, $3 \mathrm{H}), 1.32(\mathrm{~s}, 3 \mathrm{H}), 1.26(\mathrm{~d}, J=3.1 \mathrm{~Hz}, 1 \mathrm{H}) \mathrm{ppm}$.

${ }^{13}$ C NMR (150 MHz, $\left.\mathbf{C D C l}_{3}\right): \delta 165.2,157.7,137.7,129.9,122.3,121.5,115.8,115.8,106.2,77.9$, $60.5,59.1,39.7,30.6,30.5,26.5,24.8,23.0,17.8 \mathrm{ppm}$.

HRMS (ESI): calculated for $\mathrm{C}_{19} \mathrm{H}_{21} \mathrm{NO}_{3}[\mathrm{M}+\mathrm{H}]^{+} 312.1555$, found 312.1594 . 

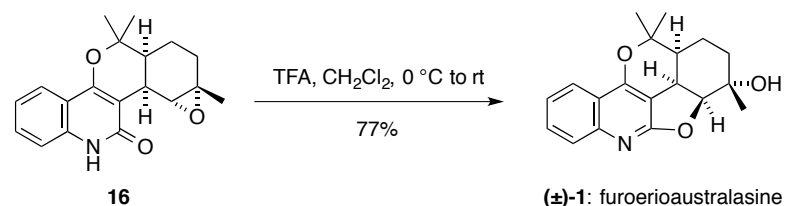

( \pm -1: furoerioaustralasine

To a solution of epoxide 16 (38.8 mg, $0.125 \mathrm{mmol}, 1.0$ equiv.) in $\mathrm{CH}_{2} \mathrm{Cl}_{2}(10 \mathrm{~mL})$ at $0{ }^{\circ} \mathrm{C}$ was added TFA (one drop). The reaction mixture was stirred for $3 \mathrm{~h}$, then warmed to room temperature and stirred for a further $20 \mathrm{~h}$ and quenched with sat. $\mathrm{NaHCO}_{3(\mathrm{aq})}(20 \mathrm{~mL})$. The organic layer was separated, and the aqueous layer was extracted with $\mathrm{CH}_{2} \mathrm{Cl}_{2}(2 \times 30 \mathrm{~mL})$. The combined extracts were then washed with brine $(100 \mathrm{~mL})$, dried over anhydrous $\mathrm{MgSO}_{4}$, filtered and concentrated in vacuo to give furoerioaustralasine (1) as a white solid (29.9 mg, $0.096 \mathrm{mmol}, 77 \%)$. Crystallization for single $\mathrm{X}$-ray diffraction was performed via vapour diffusion from $\mathrm{CHCl}_{3}$ using $\mathrm{MeOH}$ as the anti-solvent to afford rod shaped crystals. Data matched that previously reported. ${ }^{2}$

\section{Data for 1:}

$\mathbf{R}_{\mathbf{f}}: 0.20$ (EtOAc).

MP: $214.6-216.8^{\circ} \mathrm{C}(\mathrm{EtOAc})$.

FTIR (neat): 3100, 2978, 1640, 1601, 1396, 1389, 1279, 1123, 1086, 975, 923, 834, 764, $731 \mathrm{~cm}^{-1}$.

${ }^{1}$ H NMR (600 MHz, CDCl $)$ : $\delta 7.94(\mathrm{dd}, J=8.0,1.5 \mathrm{~Hz}, 1 \mathrm{H}), 7.80(\mathrm{~d}, J=8.4 \mathrm{~Hz}, 1 \mathrm{H}), 7.56(\mathrm{t}, J=$ $7.5 \mathrm{~Hz}, 1 \mathrm{H}), 7.32$ (t, $J=7.5 \mathrm{~Hz}, 1 \mathrm{H}), 4.98(\mathrm{dd}, J=9.0,4.0 \mathrm{~Hz}, 1 \mathrm{H}), 3.83(\mathrm{dd}, J=9.2,6.3 \mathrm{~Hz}, 1 \mathrm{H})$, $2.24(\mathrm{dt}, J=10.6,7.0 \mathrm{~Hz}, 1 \mathrm{H}), 1.80-1.75(\mathrm{~m}, 1 \mathrm{H}), 1.55(\mathrm{~s}, 3 \mathrm{H}), 1.46(\mathrm{~s}, 3 \mathrm{H}), 1.46-1.32(\mathrm{~m}, 1 \mathrm{H})$, $1.36-1.31(\mathrm{~m}, 1 \mathrm{H}), 1.08(\mathrm{~s}, 3 \mathrm{H}), 1.05-0.98(\mathrm{~m}, 1 \mathrm{H}) \mathrm{ppm}$.

${ }^{13}$ C NMR (150 MHz, CDCl $)$ : $\delta$ 169.3, 156.2, 149.2, 129.6, 127.8, 123.1, 121.1, 118.4, 100.2, 90.4, $82.6,72.4,35.6,33.7,32.4,27.1,25.7,25.6,18.5 \mathrm{ppm}$.

HRMS (ESI): calculated for $\mathrm{C}_{19} \mathrm{H}_{21} \mathrm{NO}_{3}[\mathrm{M}+\mathrm{H}]^{+} 312.1594$, found 312.1598.

\footnotetext{
${ }^{2}$ da Cunha, E. V. L.; Armstrong, J. A.; Gray, A. I.; Hockless, D. C. R.; Waterman, P. G.; White, A. H. Aust. J. Chem. 1993, 46, 1507.
} 

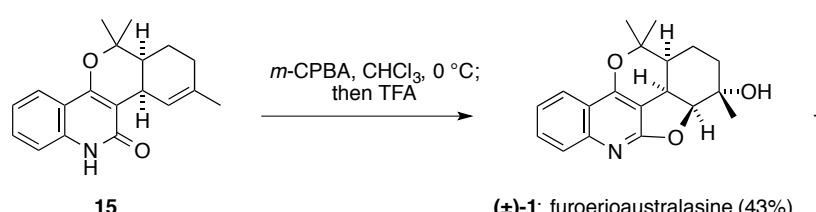

( \pm -1: furoerioaustralasine (43\%)

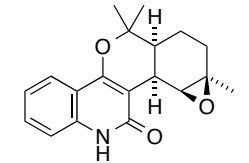

$17(21 \%)$

To alkene 15 (109 mg, $0.370 \mathrm{mmol}, 1.0$ equiv.) in $\mathrm{CHCl}_{3}(30 \mathrm{~mL})$ at $0{ }^{\circ} \mathrm{C}$ was added $m$-CPBA (114 $\mathrm{mg}, 0.508 \mathrm{mmol}, 1.4$ equiv.) and the reaction mixture was stirred at $0{ }^{\circ} \mathrm{C}$ for $3 \mathrm{~h}$. TFA (four drops) was then added, and after a further $2 \mathrm{~h}$ stirring at $0{ }^{\circ} \mathrm{C}$ the reaction was quenched with sat. $\mathrm{NaHCO}_{3(\mathrm{aq})}$ $(30 \mathrm{~mL})$. The organic layer was separated, and the aqueous layer was extracted with $\mathrm{CH}_{2} \mathrm{Cl}_{2}(2 \times 30$ $\mathrm{mL})$. The combined organic extracts were then washed with sat. $\mathrm{NaHCO}_{3}(60 \mathrm{~mL})$ and brine $(60 \mathrm{~mL})$, dried over anhydrous $\mathrm{MgSO}_{4}$, filtered and concentrated in vacuo. Purification via flash chromatography (EtOAc) followed by recrystallization from EtOAc afforded furoerioaustralasine (1) as a white solid (49.4 mg, $0.159 \mathrm{mmol}, 43 \%$ ), whilst the filtrate was concentrated to give $\mathbf{1 7}$ as a white solid (24.5 mg, $0.079 \mathrm{mmol}, 21 \%$ ). Data for $\mathbf{1}$ and $\mathbf{1 7}$ matched that previously reported. 


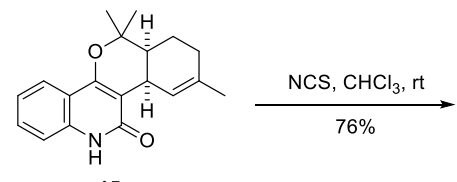

15

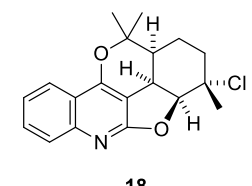

18

To a solution of alkene 15 (106 mg, $0.359 \mathrm{mmol}, 1.0$ equiv.) in $\mathrm{CHCl}_{3}(20 \mathrm{~mL})$ was added NCS (119 $\mathrm{mg}, 0.893 \mathrm{mmol}, 2.5$ equiv.) at room temperature. The reaction was stirred at room temperature for 72 $\mathrm{h}$, then quenched by addition of distilled water $(20 \mathrm{~mL})$. The organic layer was separated and the aqueous layer was extracted with $\mathrm{CH}_{2} \mathrm{Cl}_{2}(2 \times 20 \mathrm{~mL})$. The combined organic extracts were washed with brine $(50 \mathrm{~mL})$, dried over $\mathrm{MgSO}_{4}$, filtered and concentrated in vacuo. Purification via flash chromatography (1:2, hexanes:EtOAc) then afforded 18 as a white solid $(89.8 \mathrm{mg}, 0.272 \mathrm{mmol}, 76 \%)$.

\section{Data for 18:}

$\mathbf{R}_{\mathbf{f}}: 0.65$ (EtOAc).

FTIR (neat): 2980, 1657, 1598, 1408, 1387, 1278, 1131, 1086, 978, 956, 805, 765, $732 \mathrm{~cm}^{-1}$.

${ }^{1}$ H NMR (600 MHz, CDCl $)$ ): $\delta 7.95(\mathrm{~d}, J=8.2 \mathrm{~Hz}, 1 \mathrm{H}), 7.79(\mathrm{~d}, J=8.4 \mathrm{~Hz}, 1 \mathrm{H}), 7.57(\mathrm{t}, J=7.6 \mathrm{~Hz}$, 1H), 7.33 (t, $J=7.6 \mathrm{~Hz}, 1 \mathrm{H}), 5.16$ (dd, $J=9.3,2.0 \mathrm{~Hz}, 1 \mathrm{H}), 3.88$ (dd, $J=9.3,5.6 \mathrm{~Hz}, 1 \mathrm{H}), 2.45$ (td, $J$ $=8.9,5.7 \mathrm{~Hz}, 1 \mathrm{H}), 1.84-1.80(\mathrm{~m}, 1 \mathrm{H}), 1.77-1.65(\mathrm{~m}, 1 \mathrm{H}), 1.61(\mathrm{~s}, 3 \mathrm{H}), 1.54(\mathrm{~s}, 3 \mathrm{H}), 1.49(\mathrm{~s}, 3 \mathrm{H})$, $1.27(\mathrm{~m}, 1 \mathrm{H}), 1.15(\mathrm{~m}, 1 \mathrm{H}) \mathrm{ppm}$.

${ }^{13}$ C NMR (150 MHz, $\left.\mathbf{C D C l}_{3}\right): \delta 168.5,157.2,149.1,129.8,127.7,123.2,121.2,118.4,99.5,87.8$, $83.2,69.4,33.4,32.8,31.5,28.0,27.1,25.4,18.0 \mathrm{ppm}$.

HRMS (ESI): calculated for $\mathrm{C}_{19} \mathrm{H}_{21} \mathrm{NO}_{2} \mathrm{Cl}[\mathrm{M}+\mathrm{H}]^{+} 330.1261$, found 330.1257. 


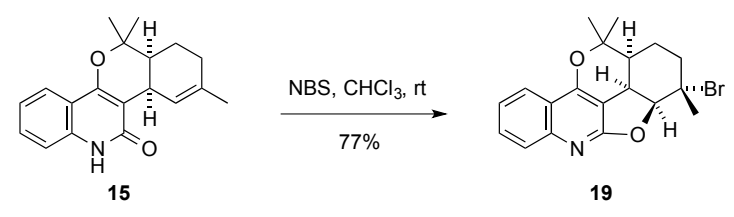

To a solution of alkene 15 (74.2 $\mathrm{mg}, 0.251 \mathrm{mmol}, 1.0$ equiv.) in $\mathrm{CHCl}_{3}(20 \mathrm{~mL})$ was added NBS (54.3 $\mathrm{mg}, 0.307 \mathrm{mmol}, 1.2$ equiv.) at room temperature. The reaction mixture was stirred at room temperature for $20 \mathrm{~min}$, then quenched by the addition of distilled water $(30 \mathrm{~mL})$. The organic layer was separated, and the aqueous layer was extracted with $\mathrm{CH}_{2} \mathrm{Cl}_{2}(2 \times 30 \mathrm{~mL})$. The combined organic extracts were washed with brine $(80 \mathrm{~mL})$, dried over $\mathrm{MgSO}_{4}$, filtered and concentrated in vacuo. Purification via flash chromatography (EtOAc) then gave 19 as a yellow oil (72.7 mg, $0.194 \mathrm{mmol}$, $77 \%)$.

\section{Data for 19:}

Rf: 0.45 (2:1, hexanes:EtOAc).

FTIR (neat): 2978, 1657, 1597, 1387, 1278, 1086, 977, 948, 802, $760 \mathrm{~cm}^{-1}$.

${ }^{1}$ H NMR (600 MHz, CDCl $)$ : $\delta 7.94(\mathrm{~d}, J=8.1 \mathrm{~Hz}, 1 \mathrm{H}), 7.79$ (d, $\left.J=8.4 \mathrm{~Hz}, 1 \mathrm{H}\right), 7.56(\mathrm{dd}, J=8.5$, $6.9 \mathrm{~Hz}, 1 \mathrm{H}), 7.32(\mathrm{dd}, J=8.2,6.9 \mathrm{~Hz}, 1 \mathrm{H}), 5.38(\mathrm{~d}, J=9.3 \mathrm{~Hz}, 1 \mathrm{H}), 3.87(\mathrm{dd}, J=9.4,5.7 \mathrm{~Hz}, 1 \mathrm{H})$, $2.55(\mathrm{ddd}, J=9.3,7.0,4.8 \mathrm{~Hz}, 1 \mathrm{H}), 1.83(\mathrm{~s}, 3 \mathrm{H}), 1.80(\mathrm{~m}, 2 \mathrm{H}), 1.54(\mathrm{~s}, 3 \mathrm{H}), 1.49$ (s, 3H), 1.26 (m, $1 \mathrm{H}), 1.19(\mathrm{~m}, 1 \mathrm{H}) \mathrm{ppm}$.

${ }^{13}$ C NMR (150 MHz, $\left.\mathbf{C D C l}_{3}\right): \delta 168.4,157.2,149.0,129.8,127.7,123.2,121.2,118.4,99.4,88.1$, $83.2,65.8,33.4,32.6,32.5,29.8,29.5,27.1,25.3,18.8 \mathrm{ppm}$.

HRMS (ESI): calculated for $\mathrm{C}_{19} \mathrm{H}_{21} \mathrm{NO}_{2} \mathrm{Br}[\mathrm{M}+\mathrm{H}]^{+} 374.0750$, found 374.0767 . 


\section{NMR spectra}

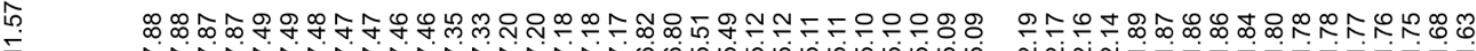

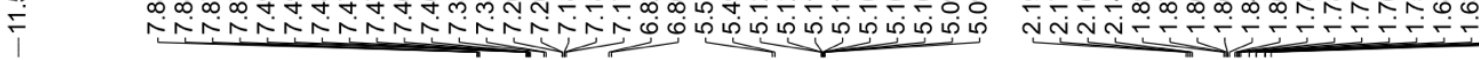

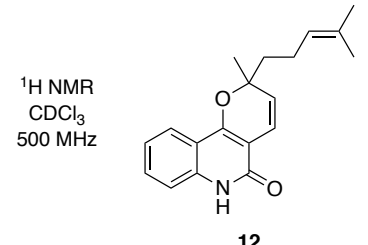

12

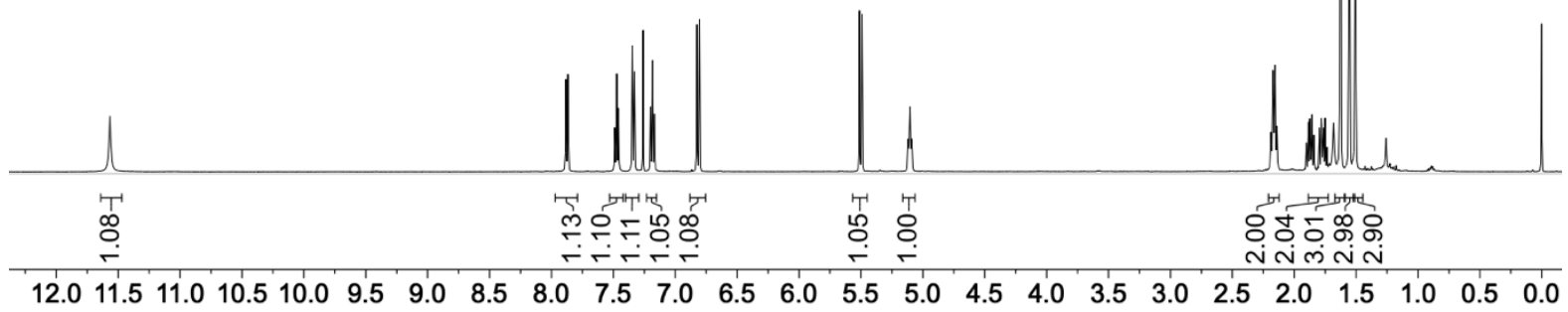

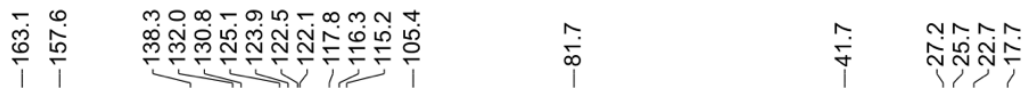

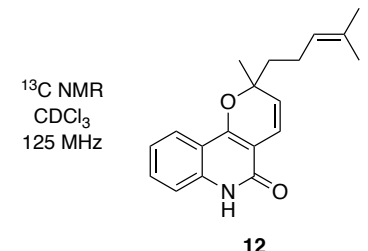

12

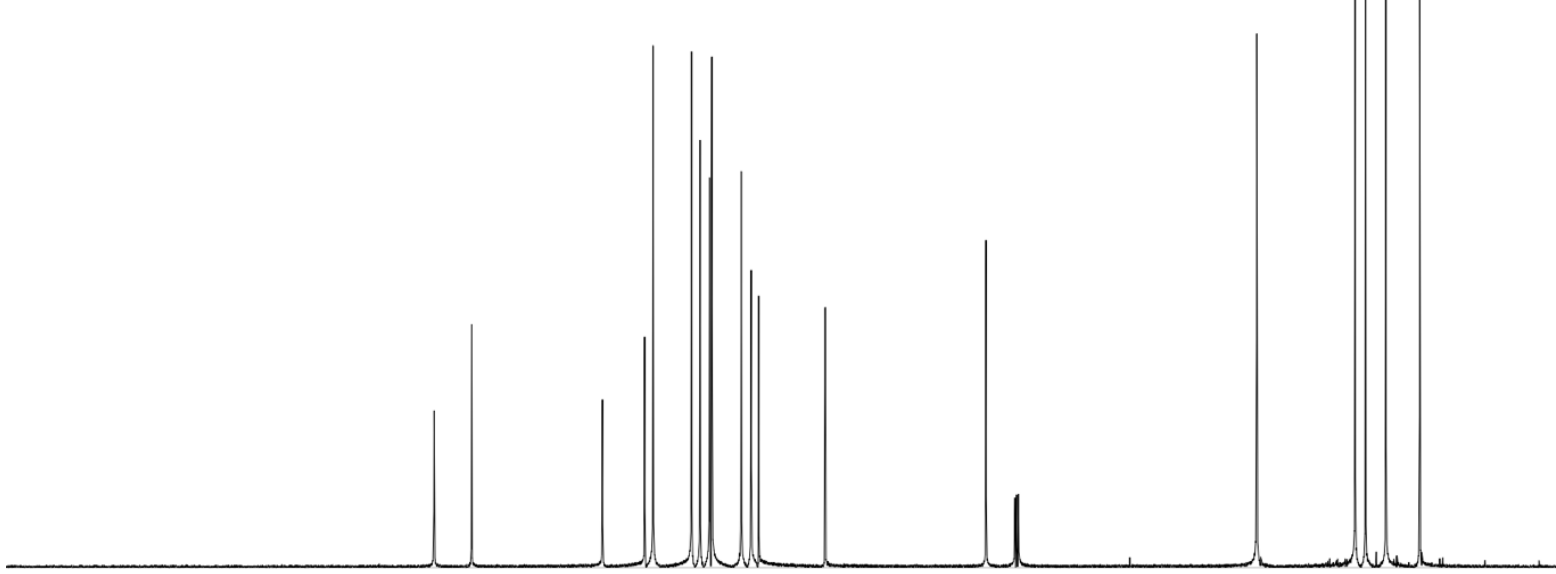

$\begin{array}{lllllllllllllllllllllll}220 & 210 & 200 & 190 & 180 & 170 & 160 & 150 & 140 & 130 & 120 & 110 & 100 & 90 & 80 & 70 & 60 & 50 & 40 & 30 & 20 & 10 & 0\end{array}$ 

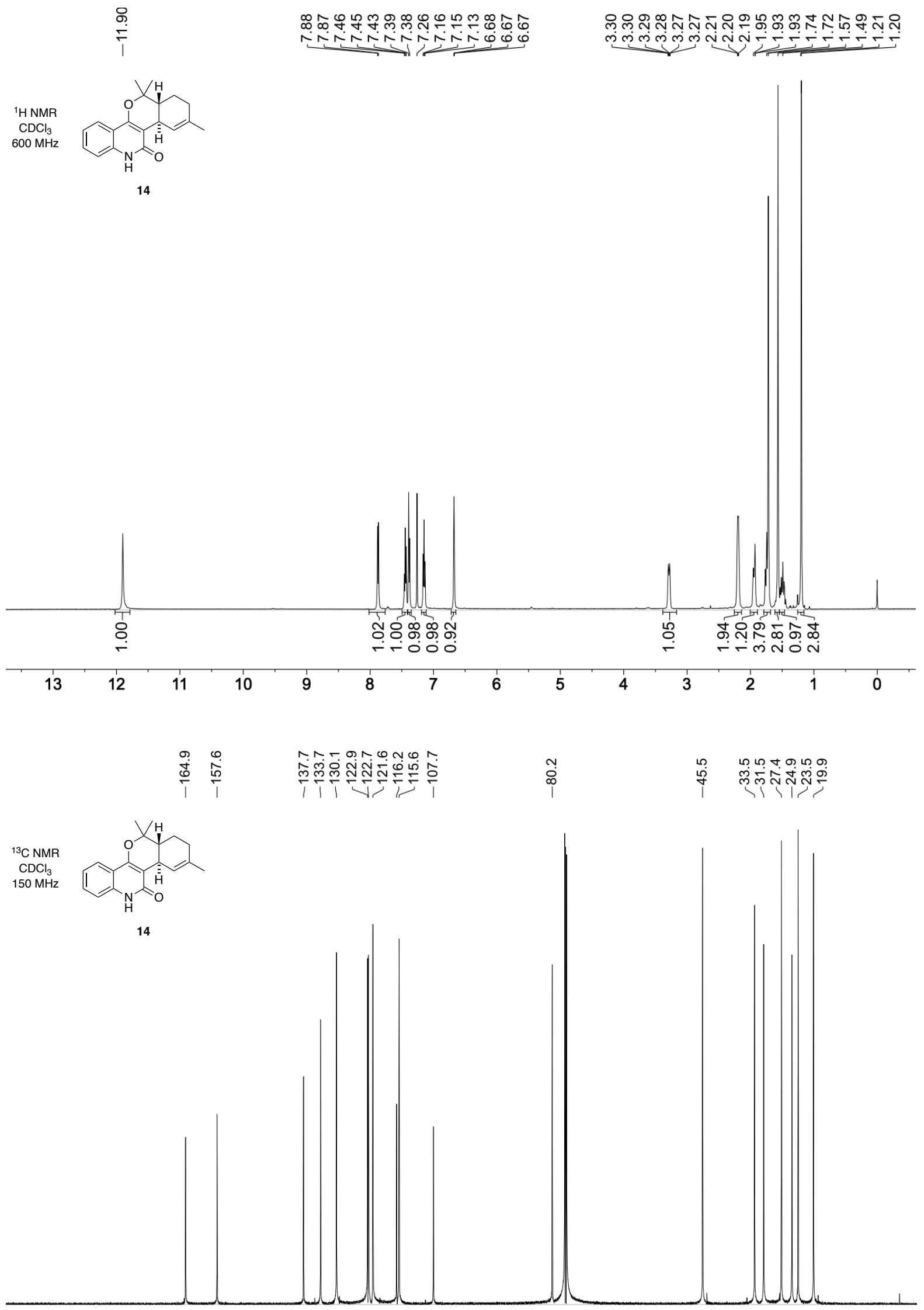

$\begin{array}{llllllllllllllllllllllllllll}200 & 190 & 180 & 170 & 160 & 150 & 140 & 130 & 120 & 110 & 100 & 90 & 80 & 70 & 60 & 50 & 40 & 30 & 20 & 10 & 0\end{array}$ 

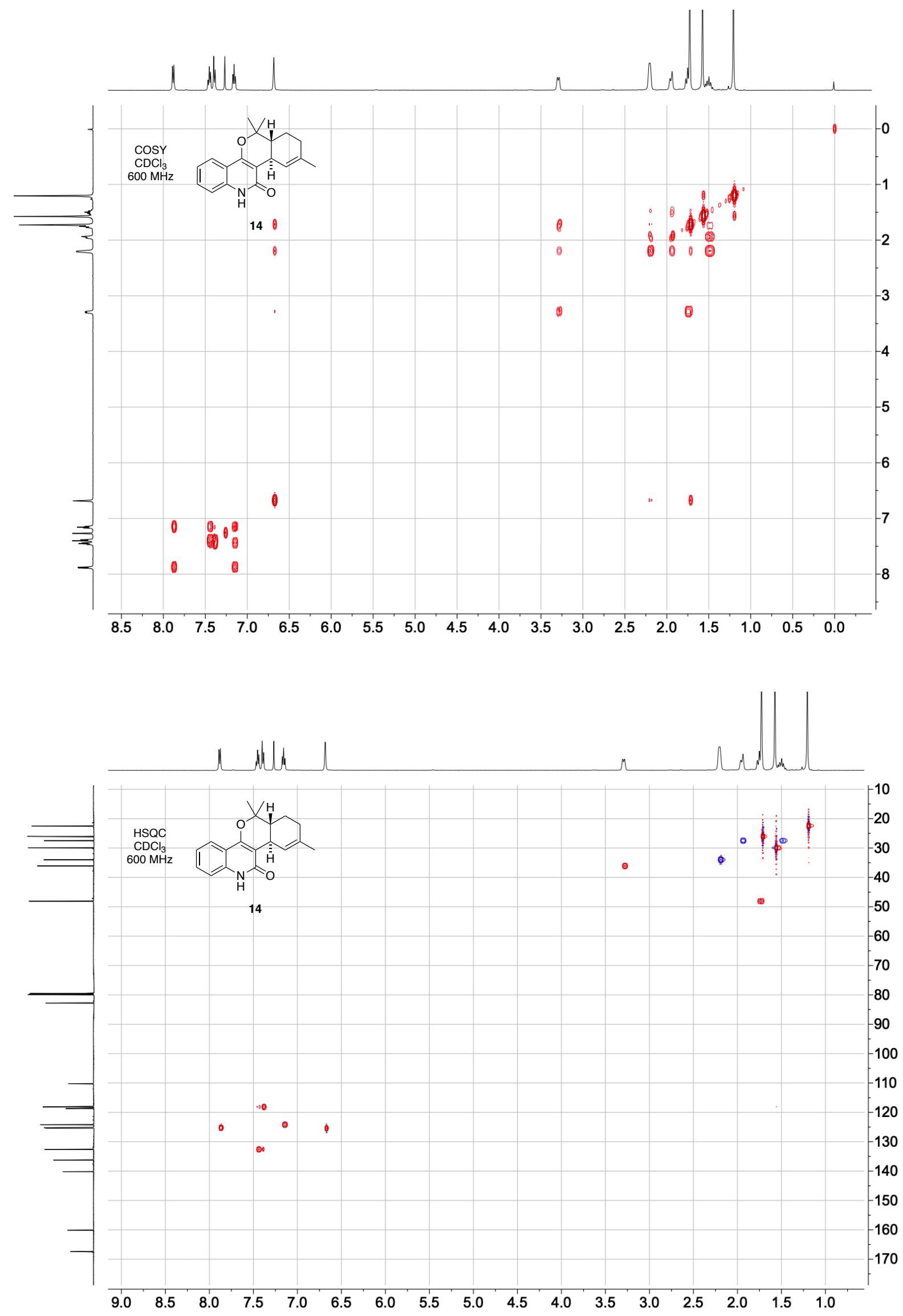

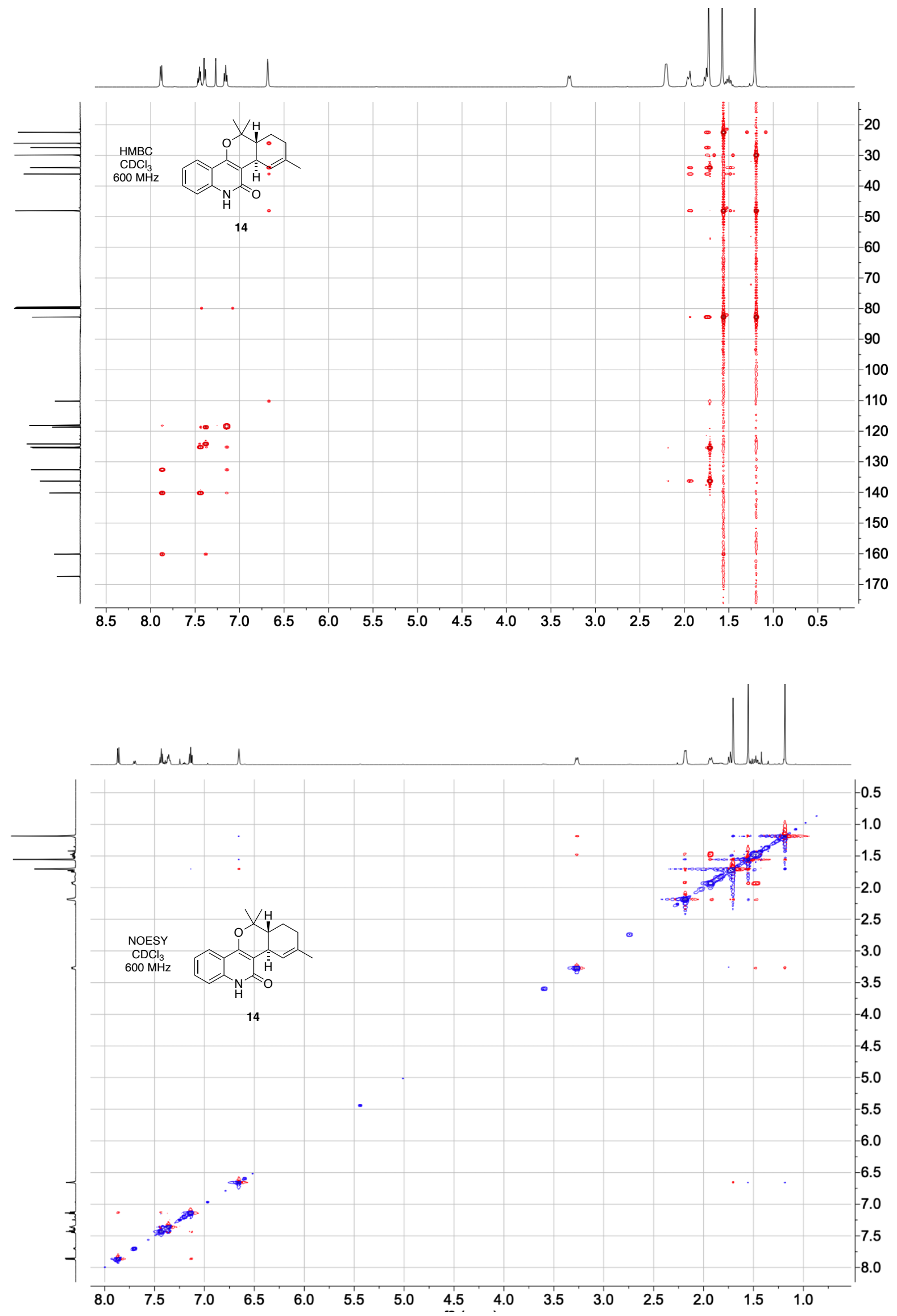


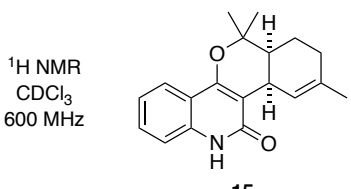

15
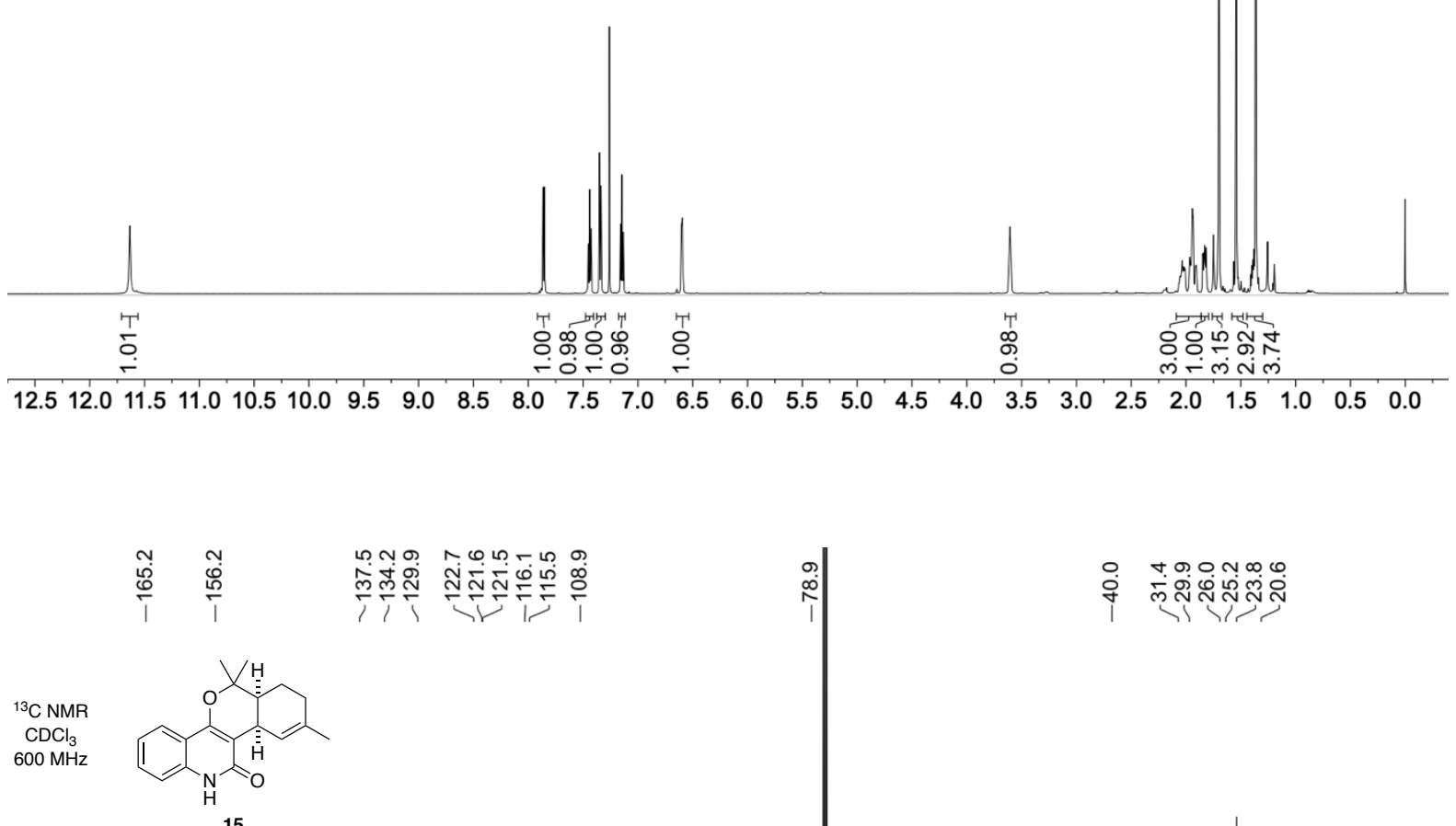

15

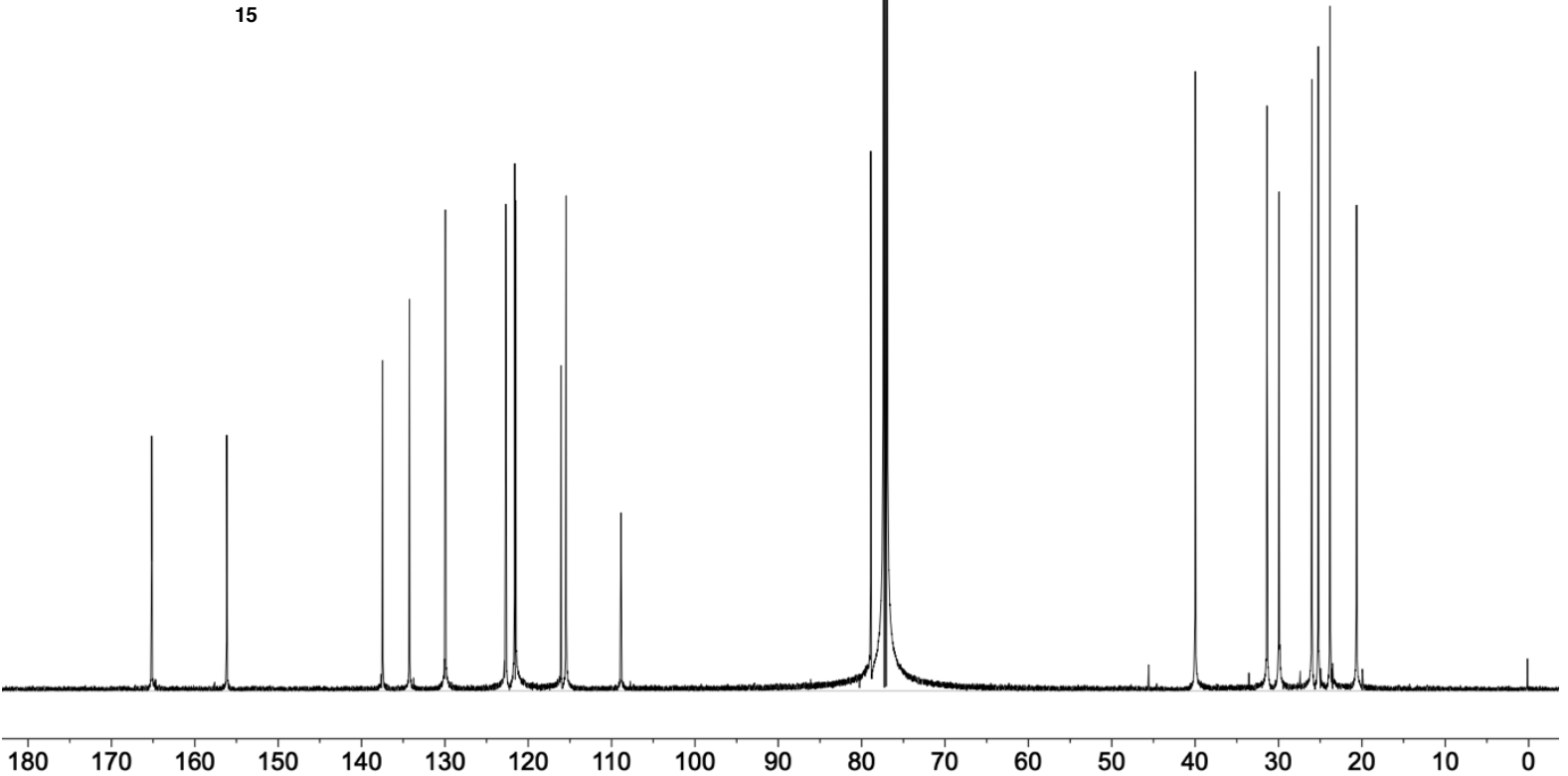



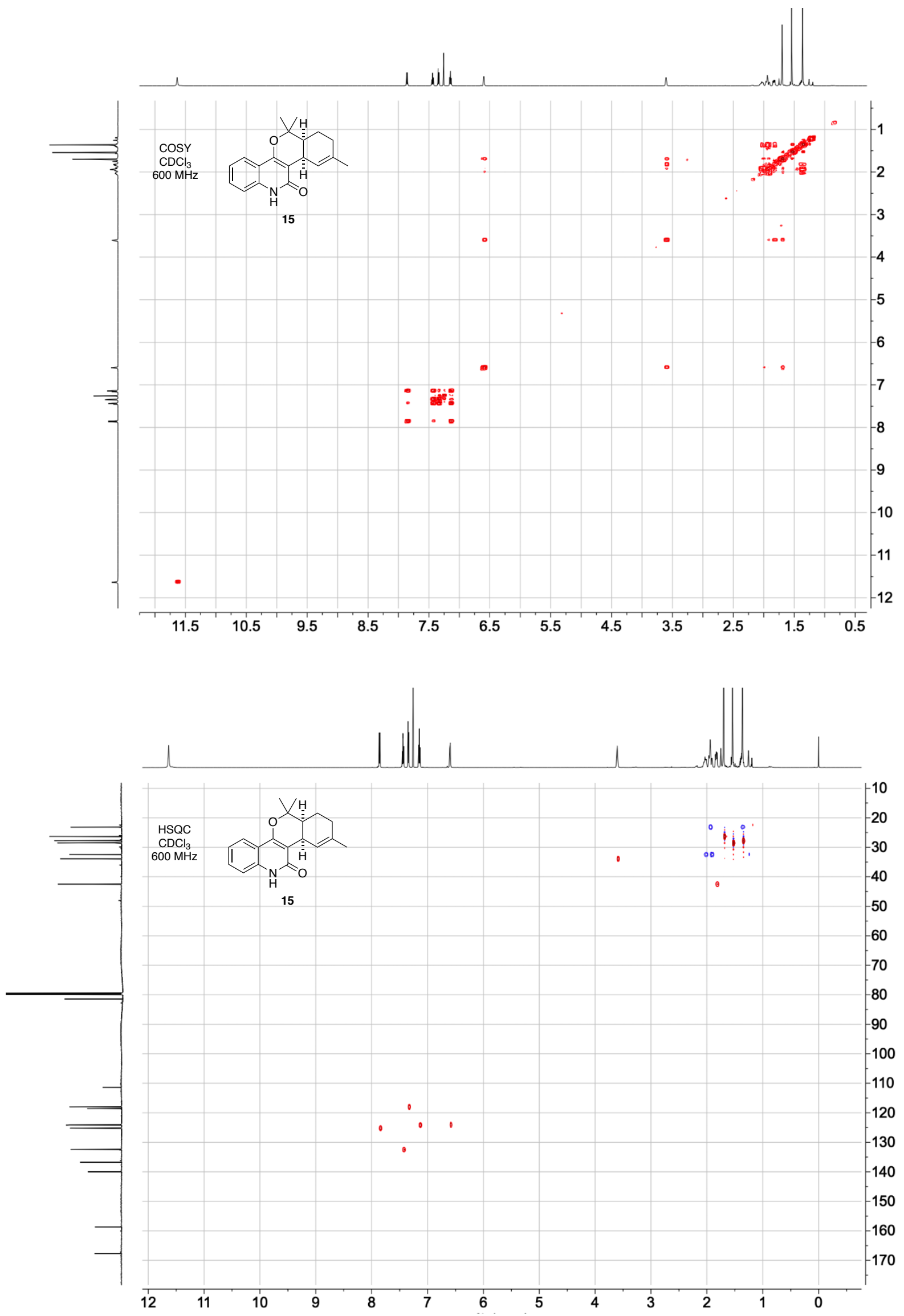

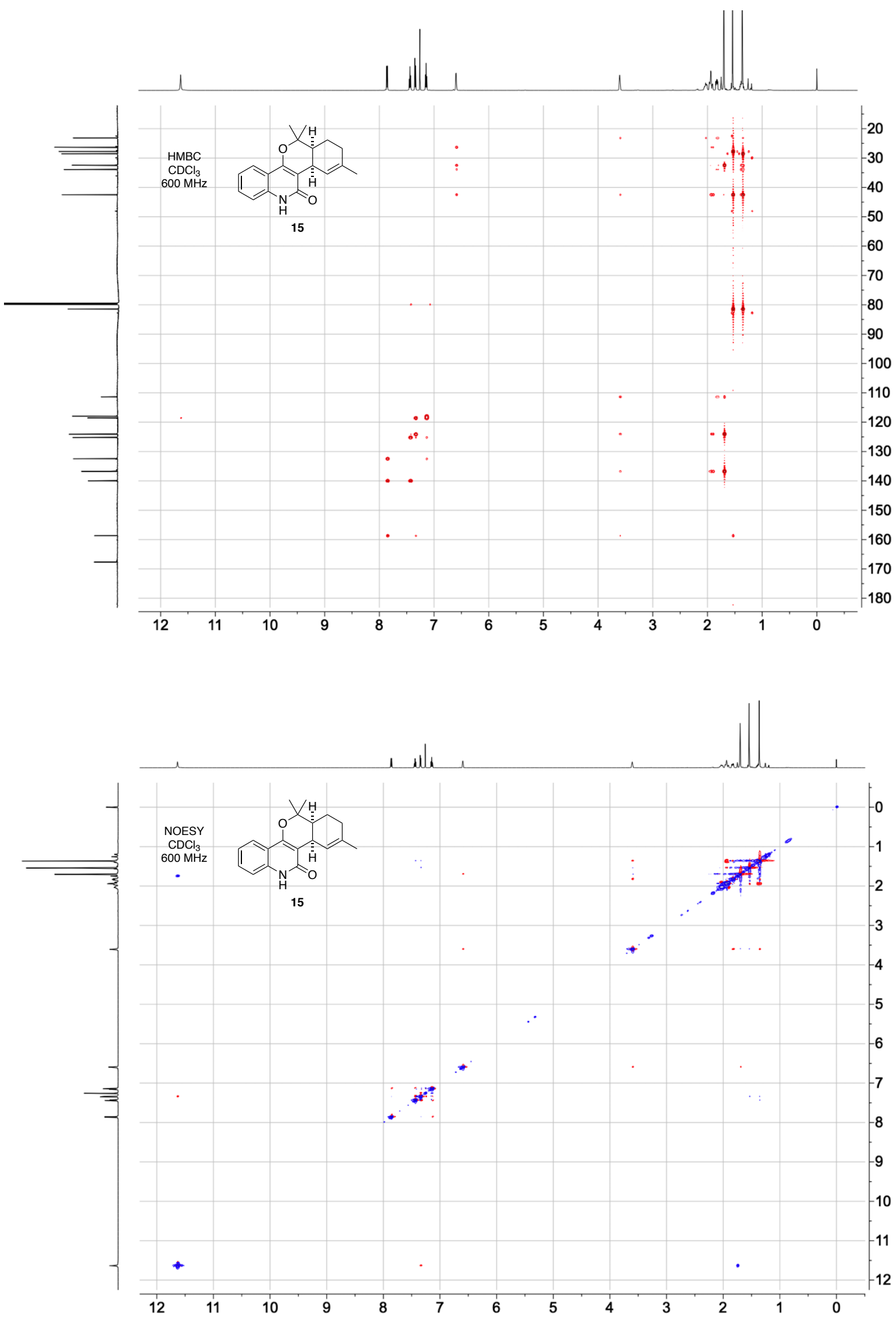


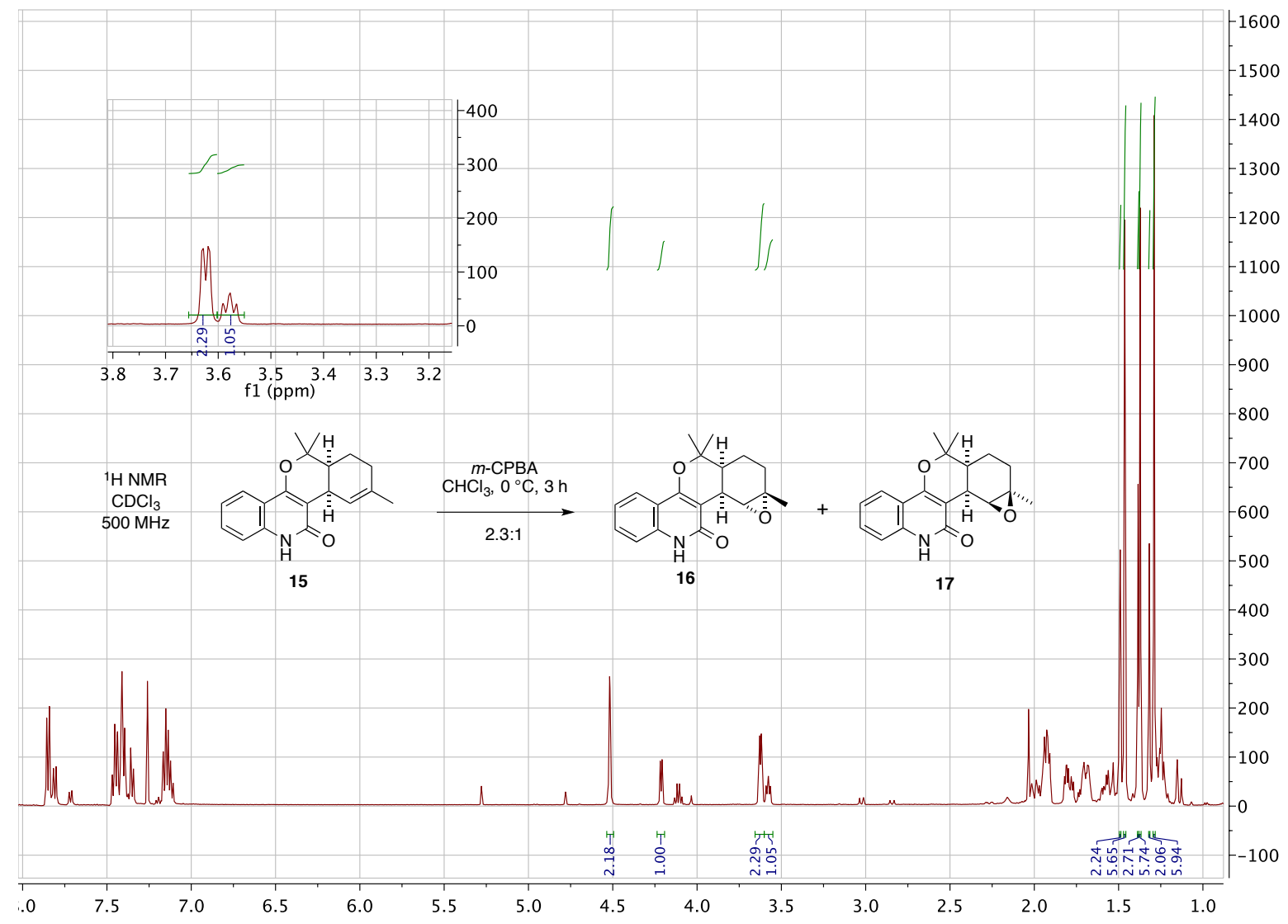




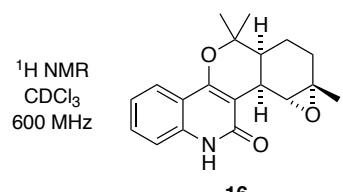

16

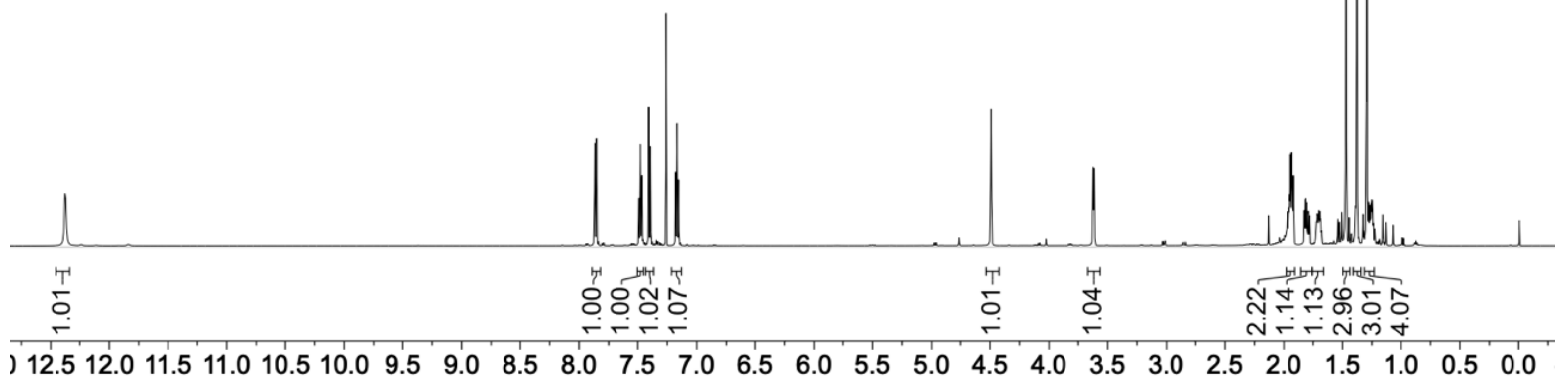

în

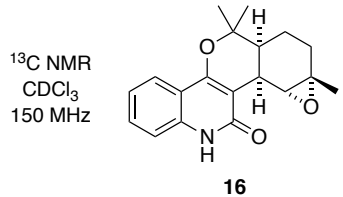

16

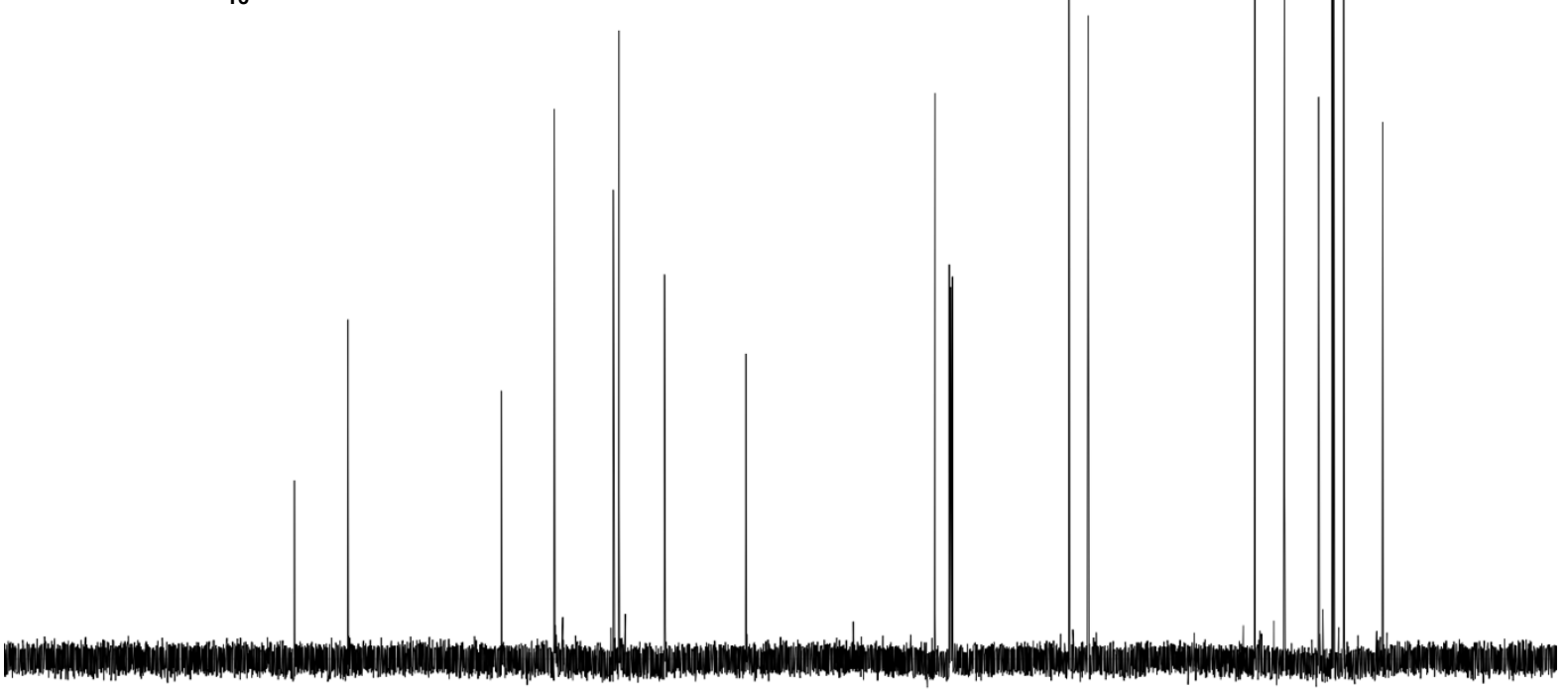

$\begin{array}{lllllllllllllllllllll}200 & 190 & 180 & 170 & 160 & 150 & 140 & 130 & 120 & 110 & 100 & 90 & 80 & 70 & 60 & 50 & 40 & 30 & 20 & 10 & 0\end{array}$ 

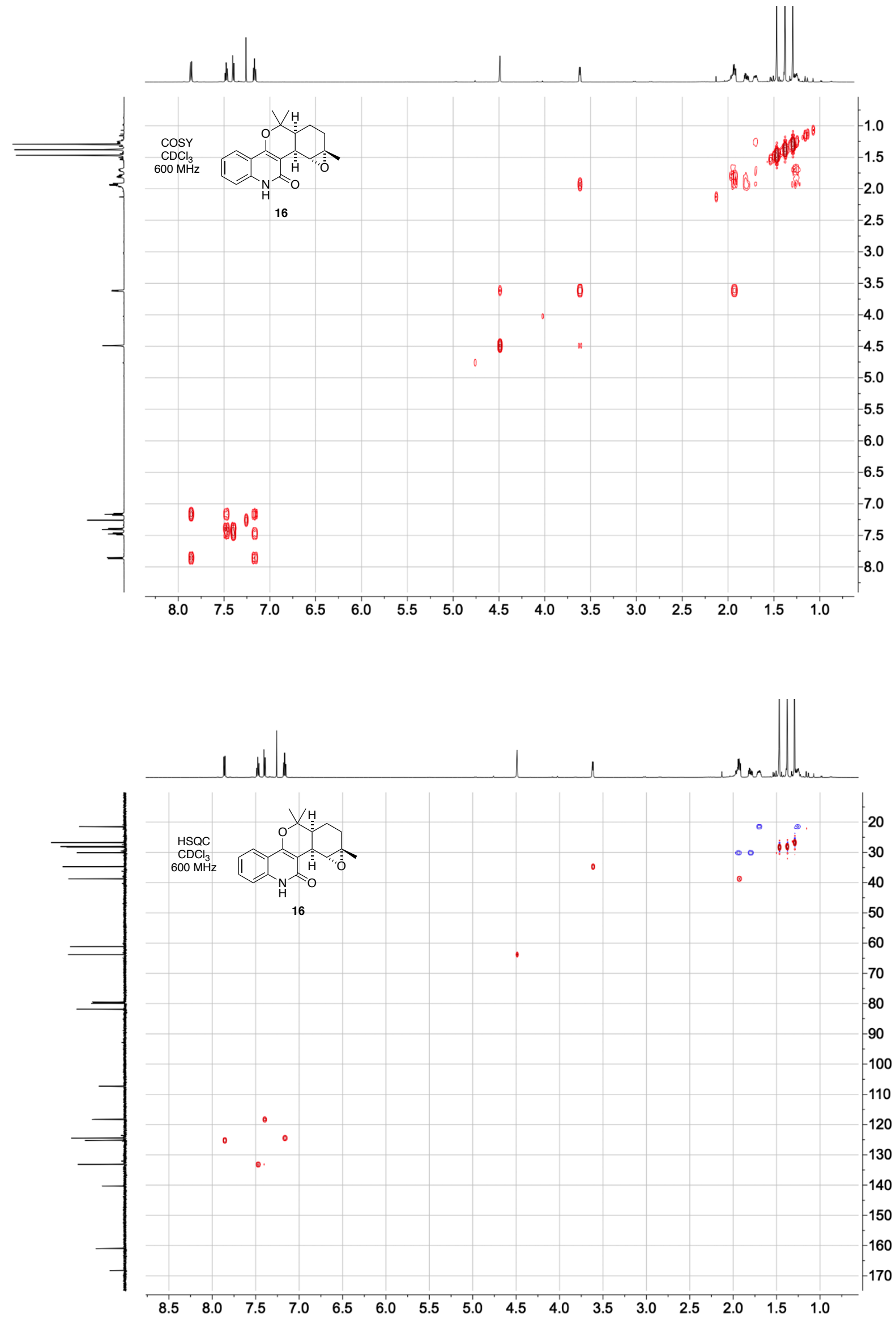

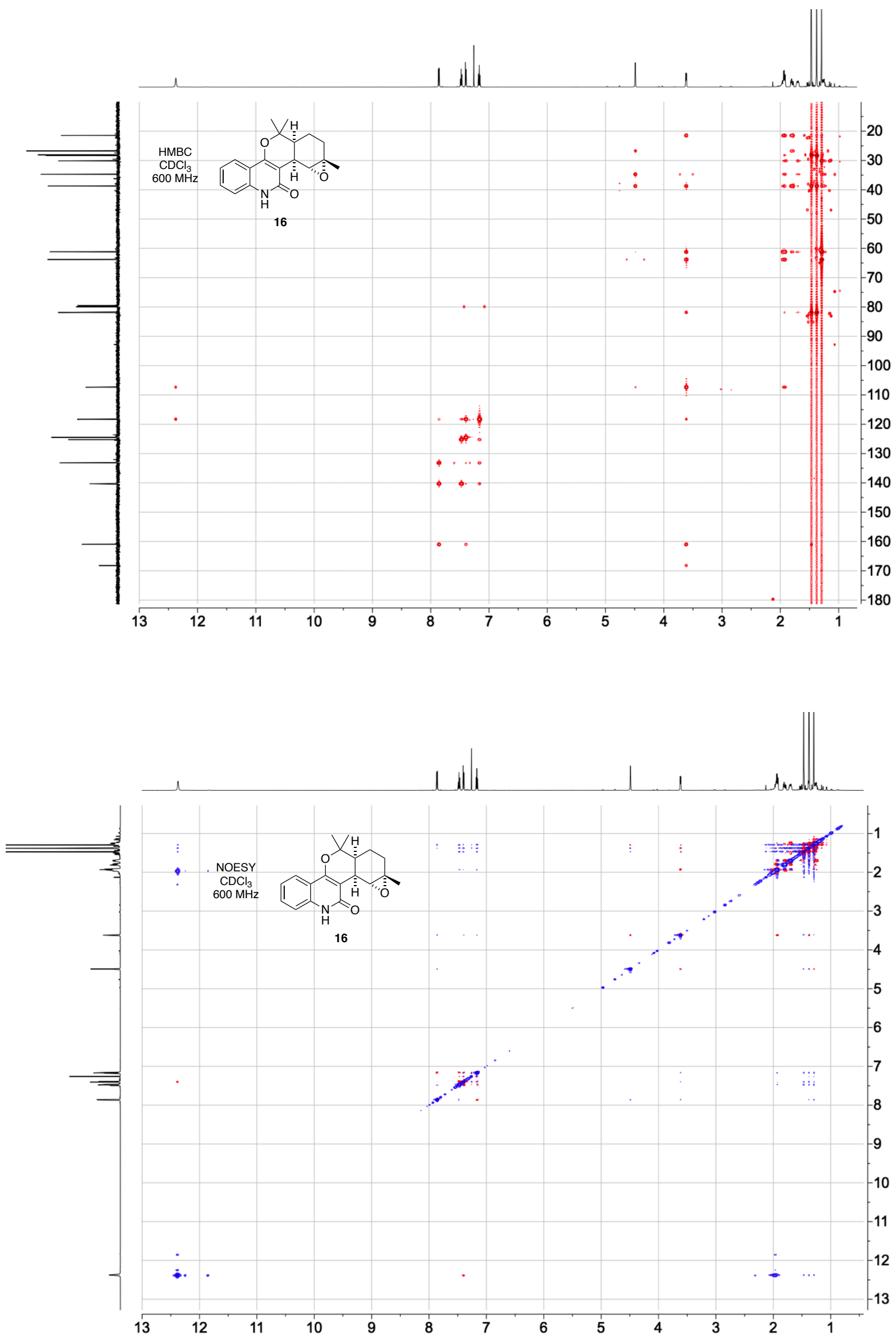


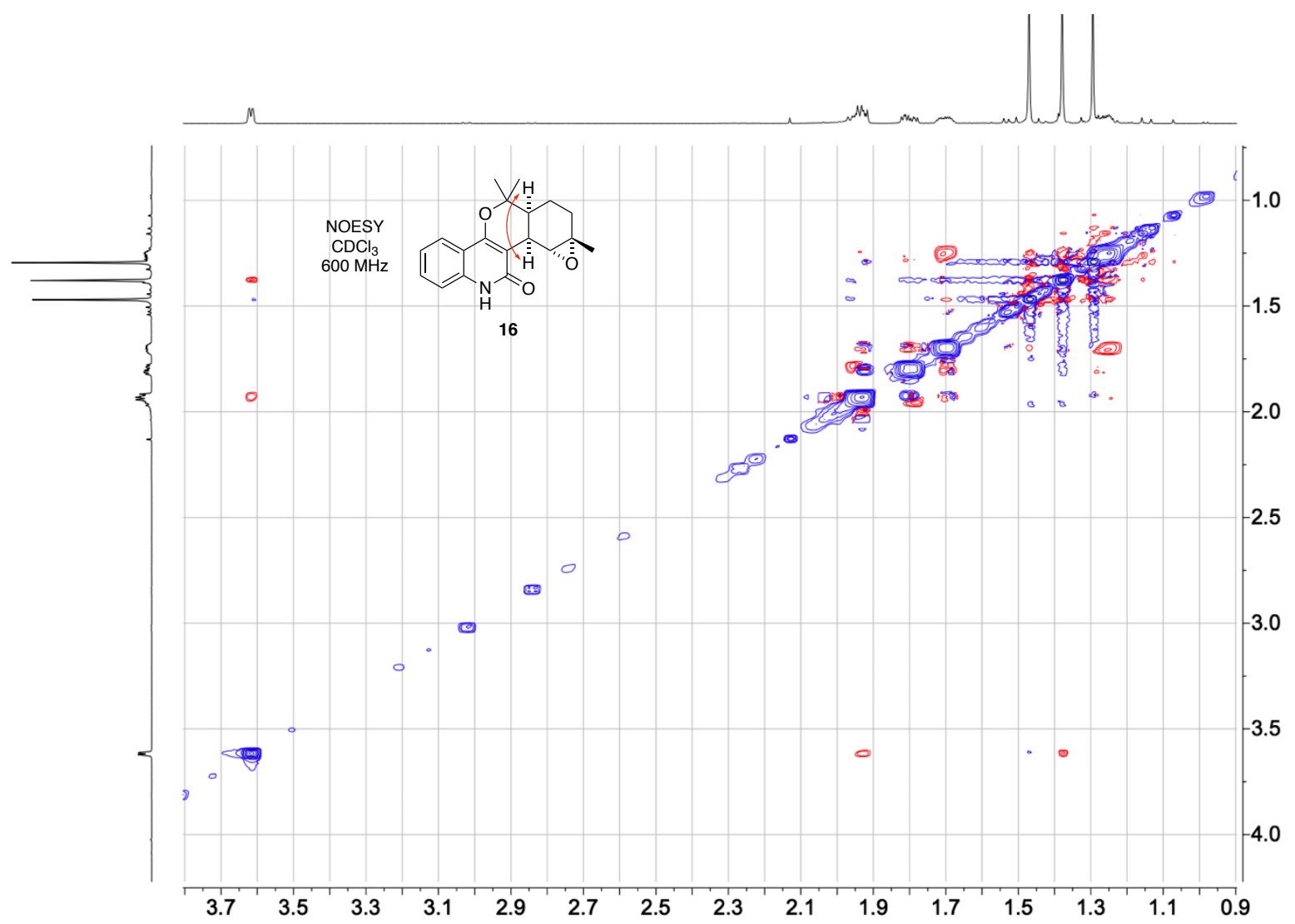



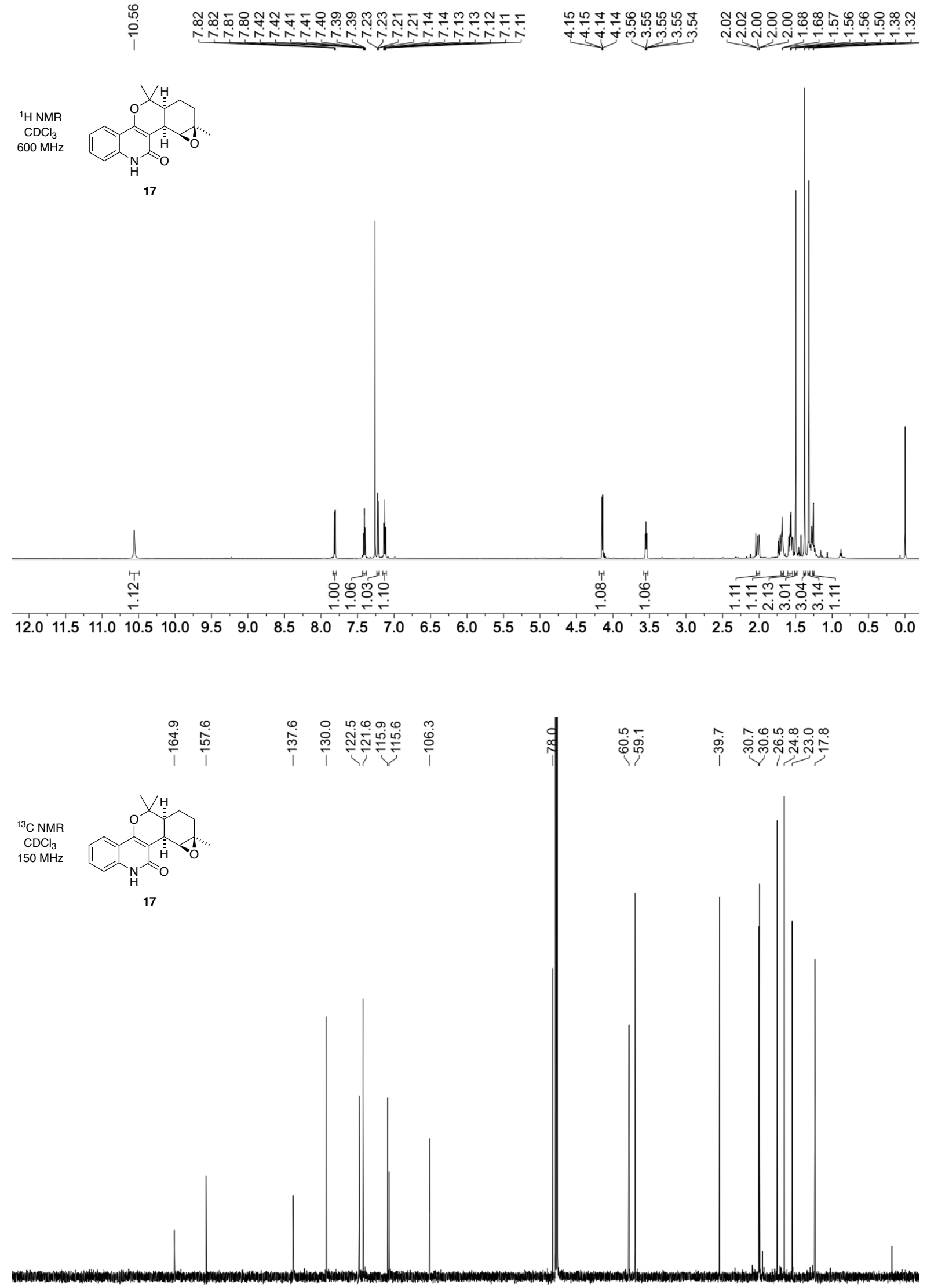

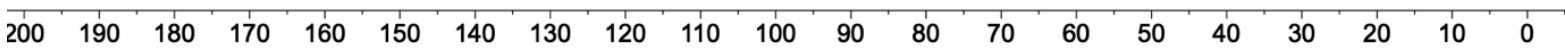



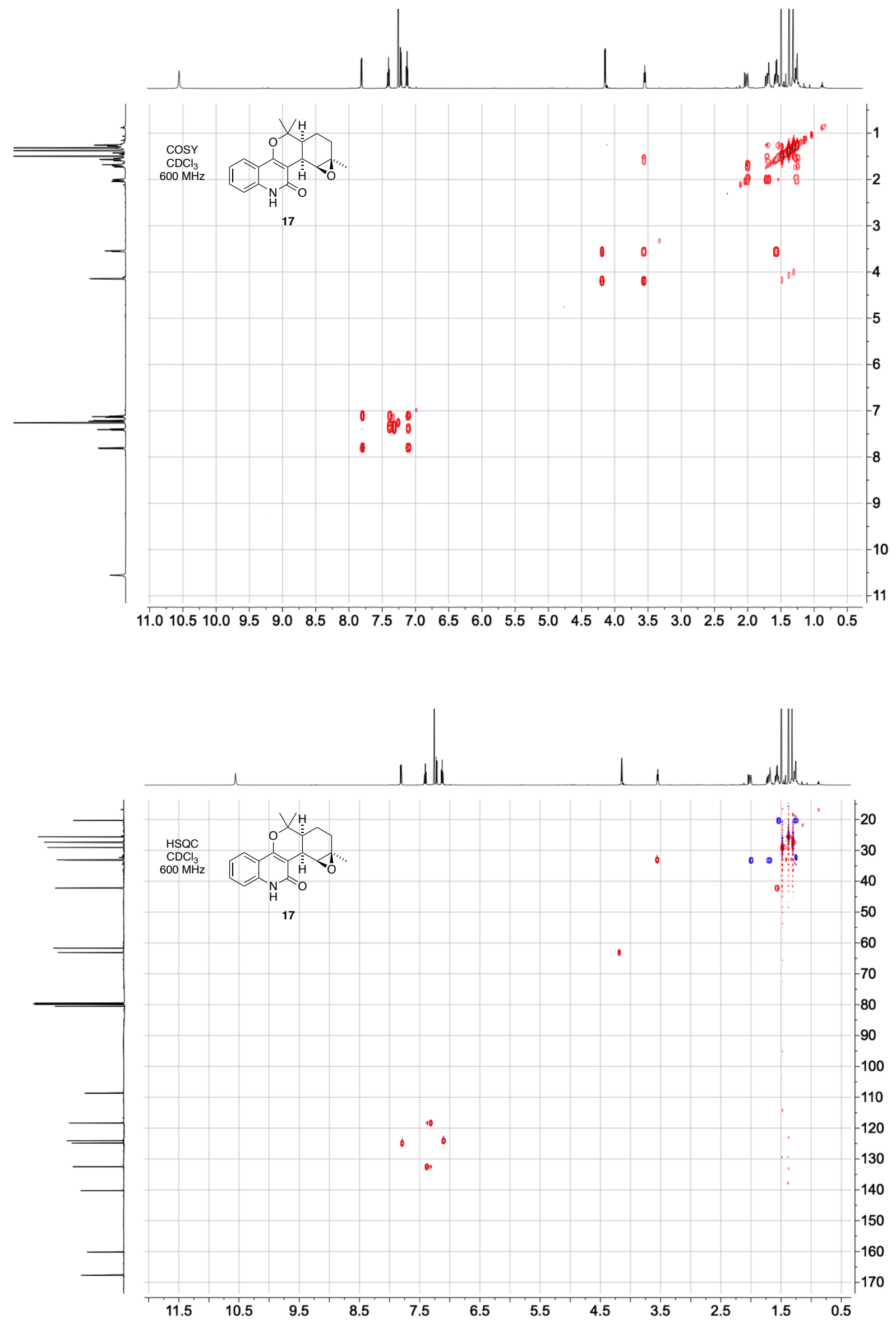

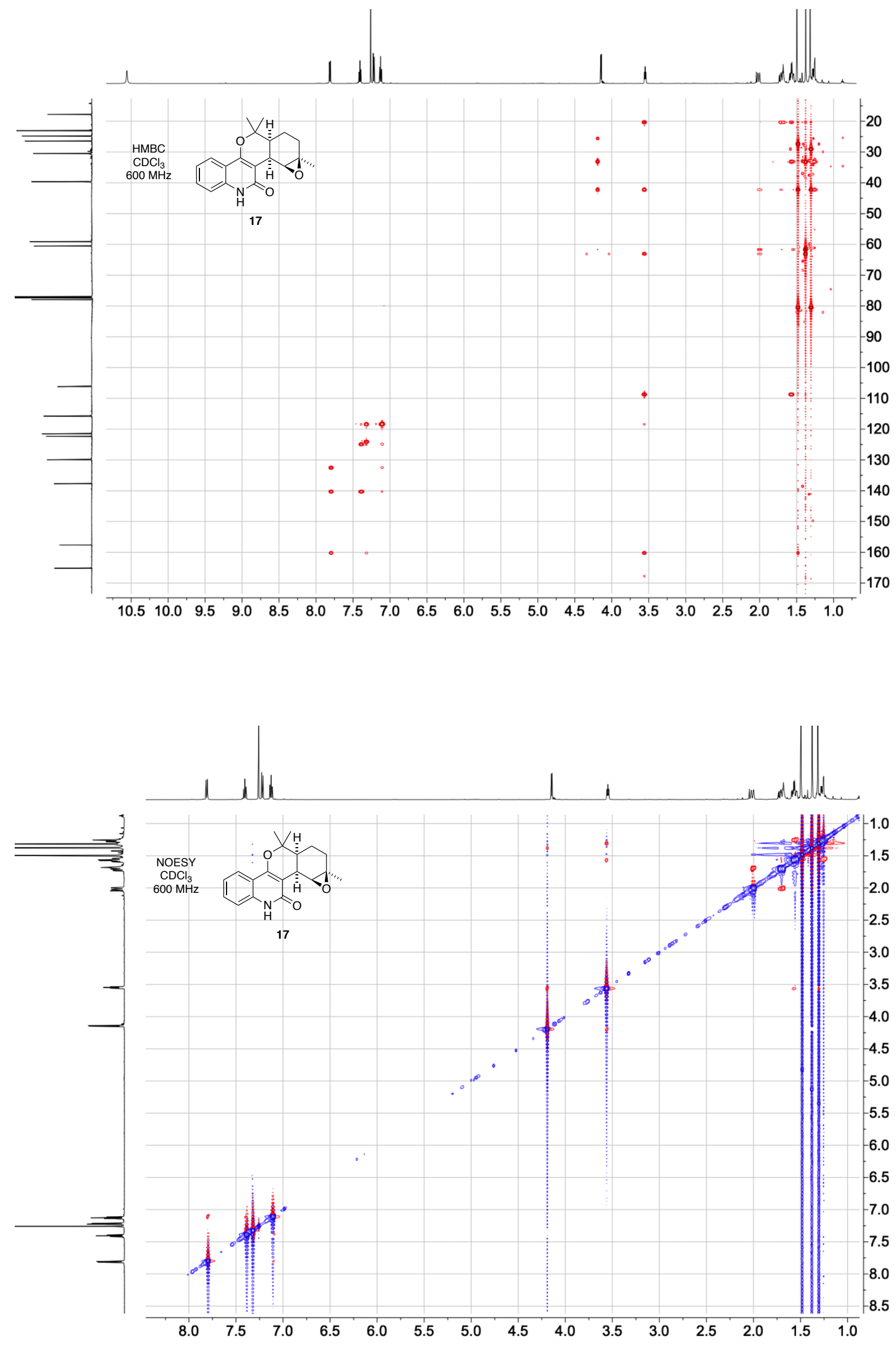


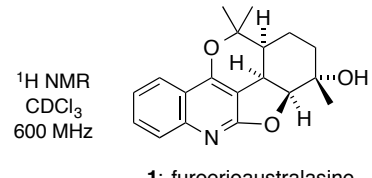

1: furoerioaustralasine
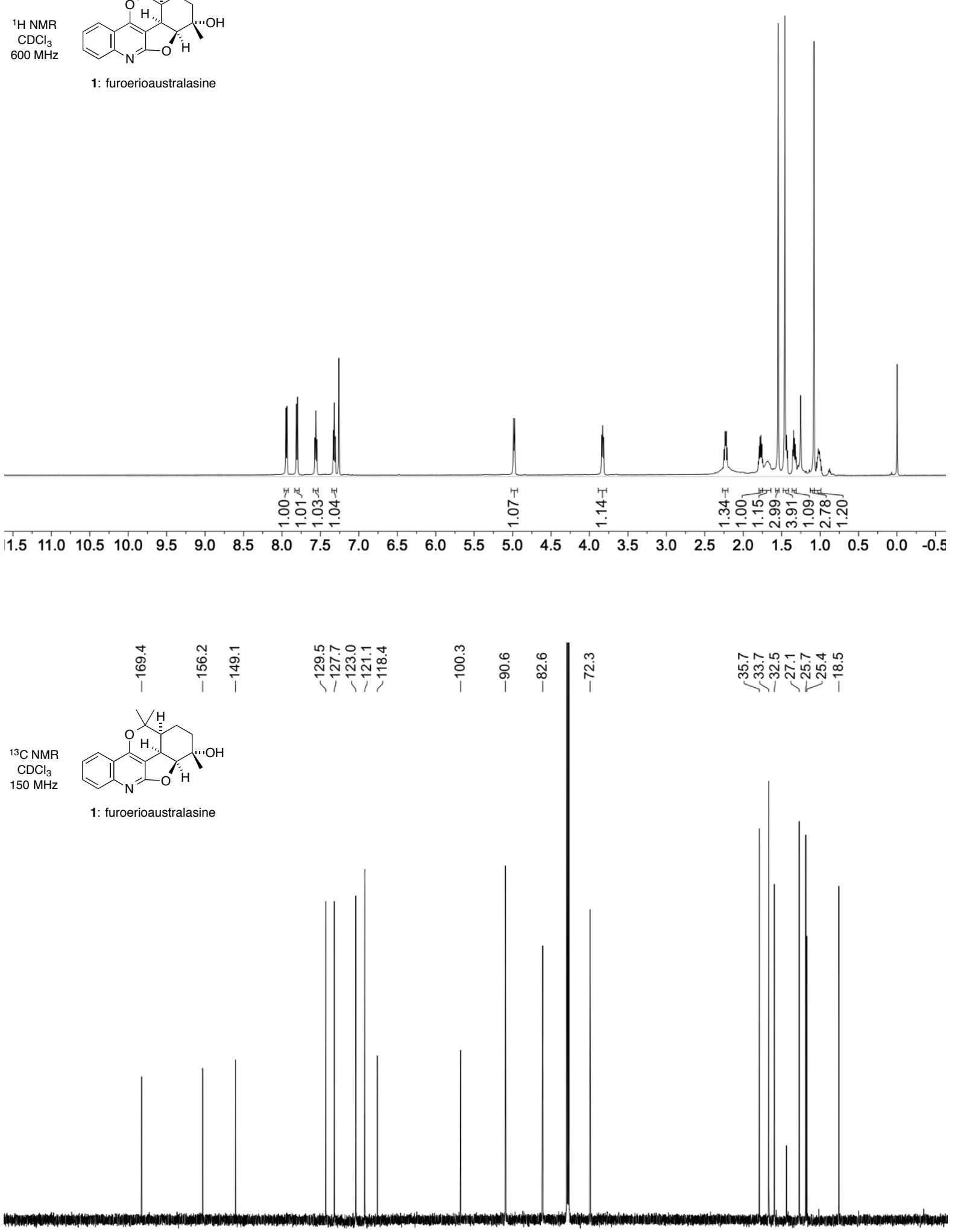

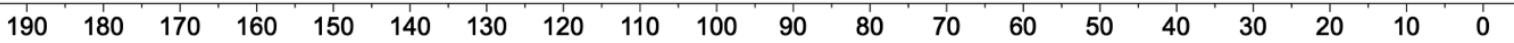



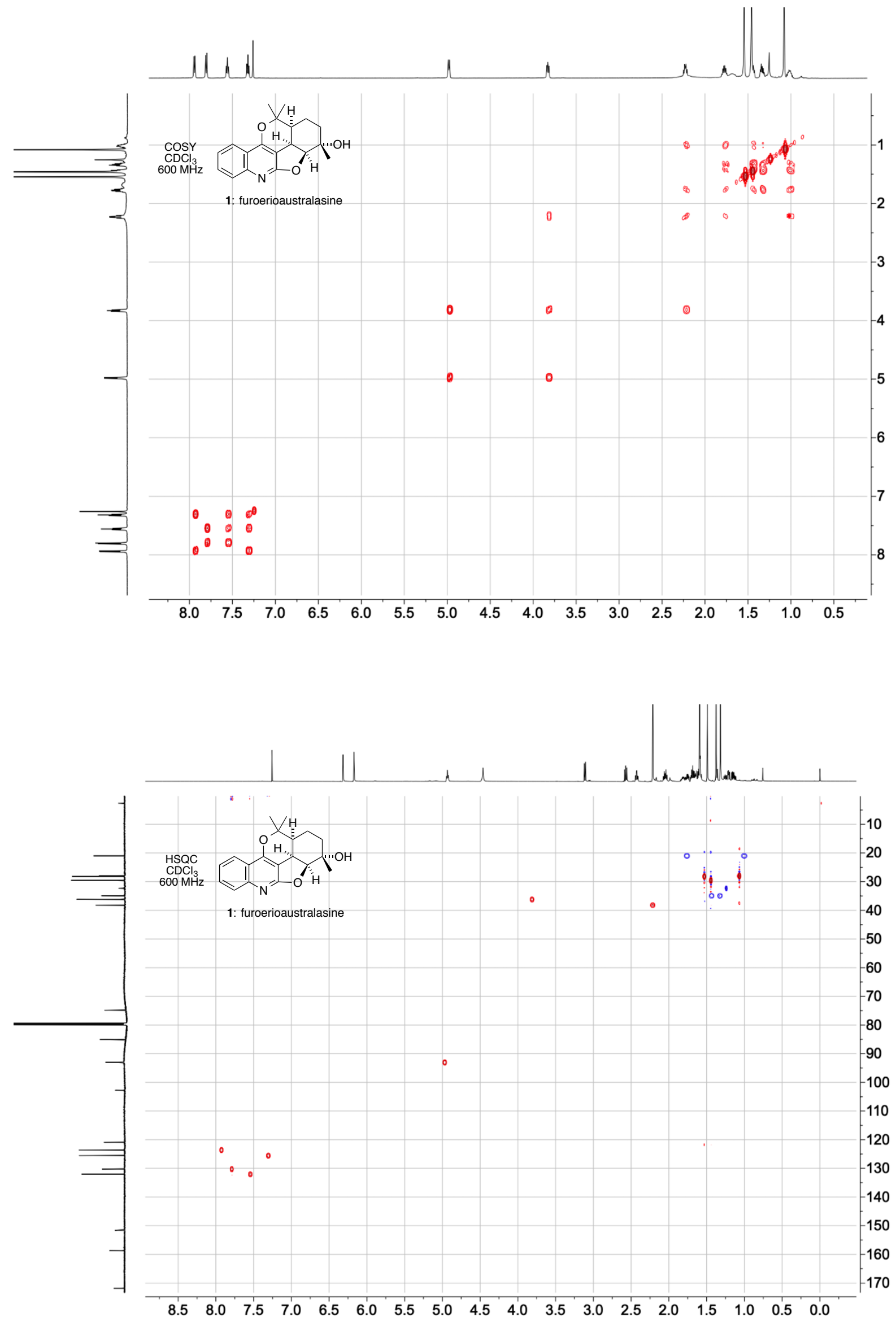

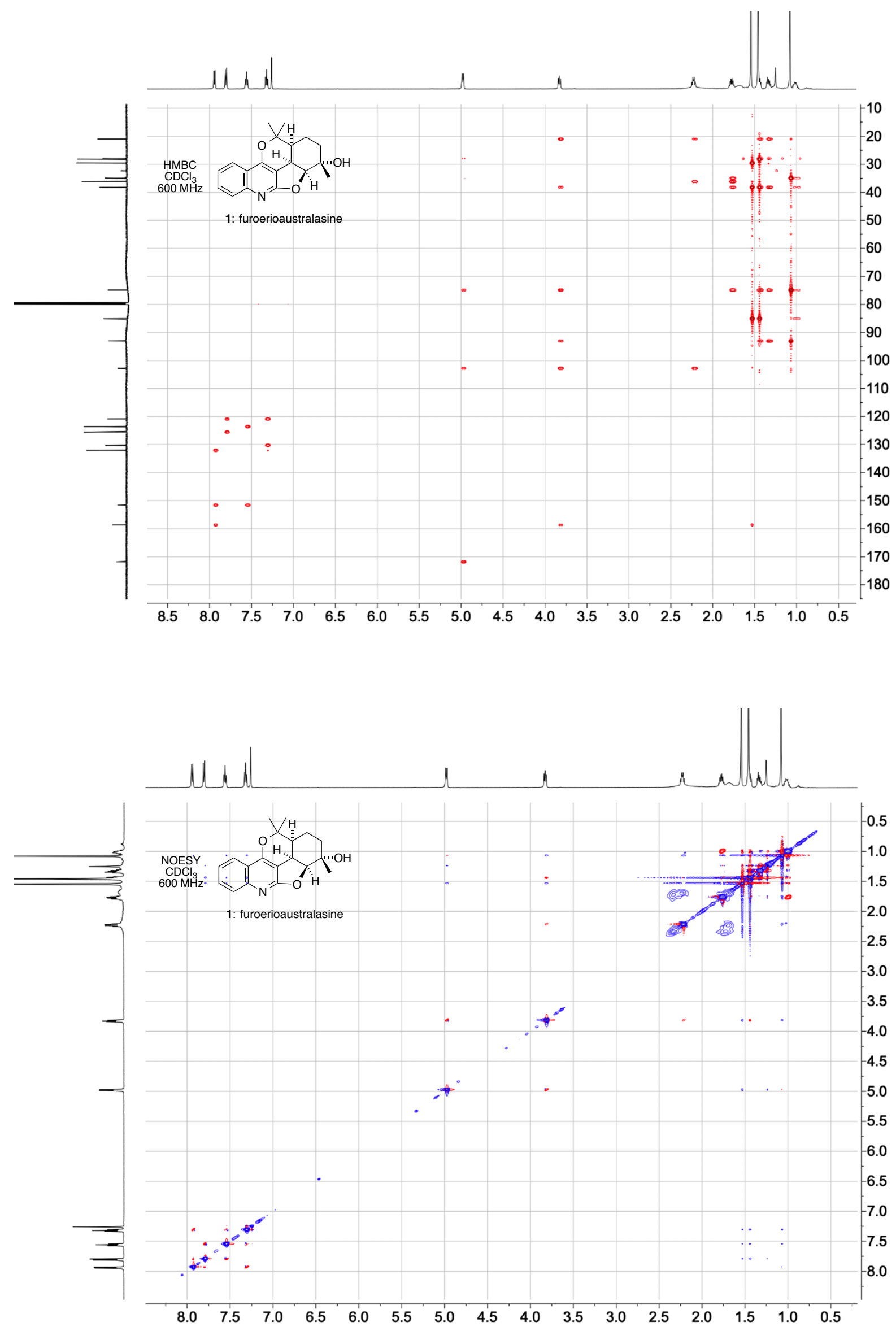

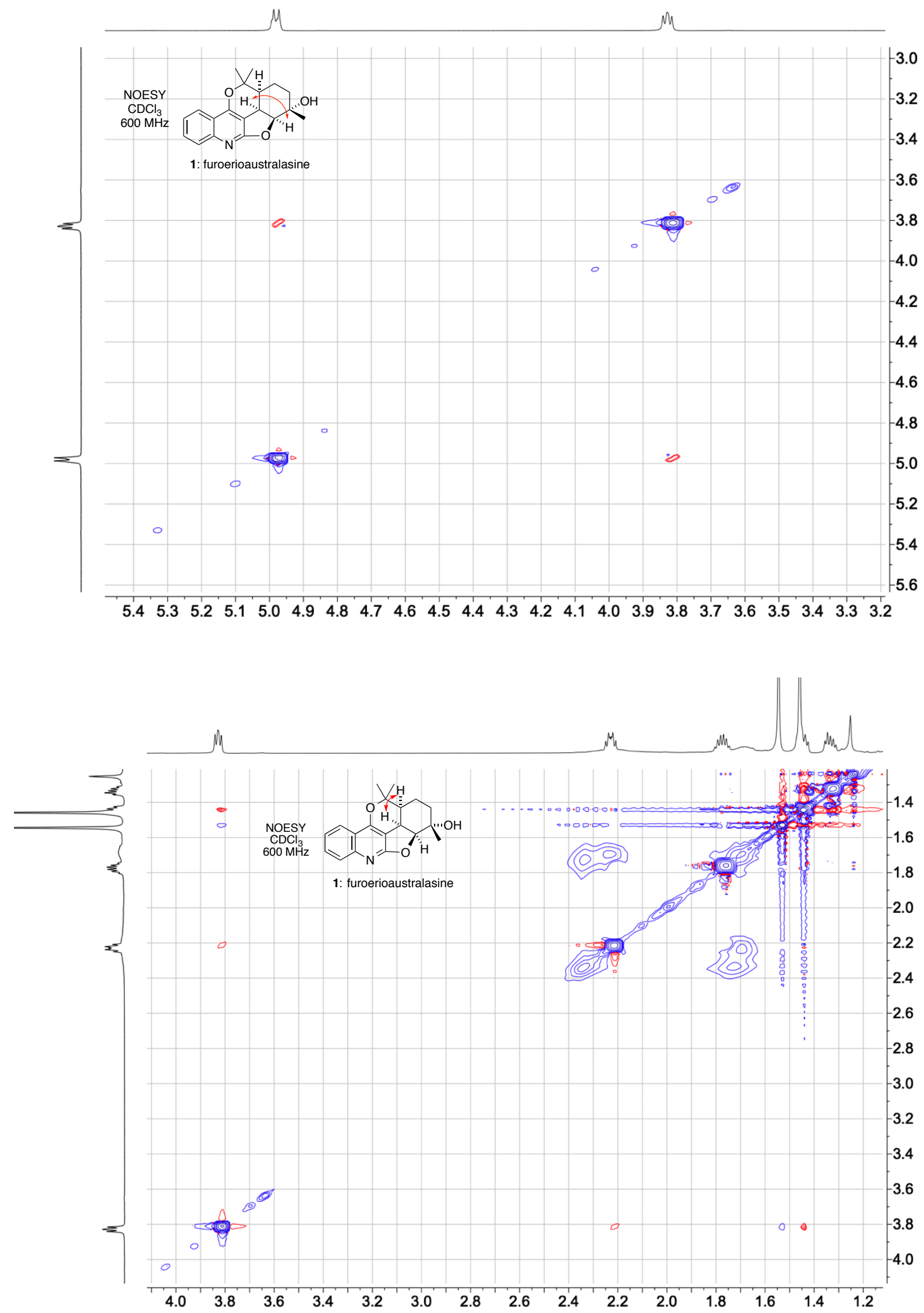

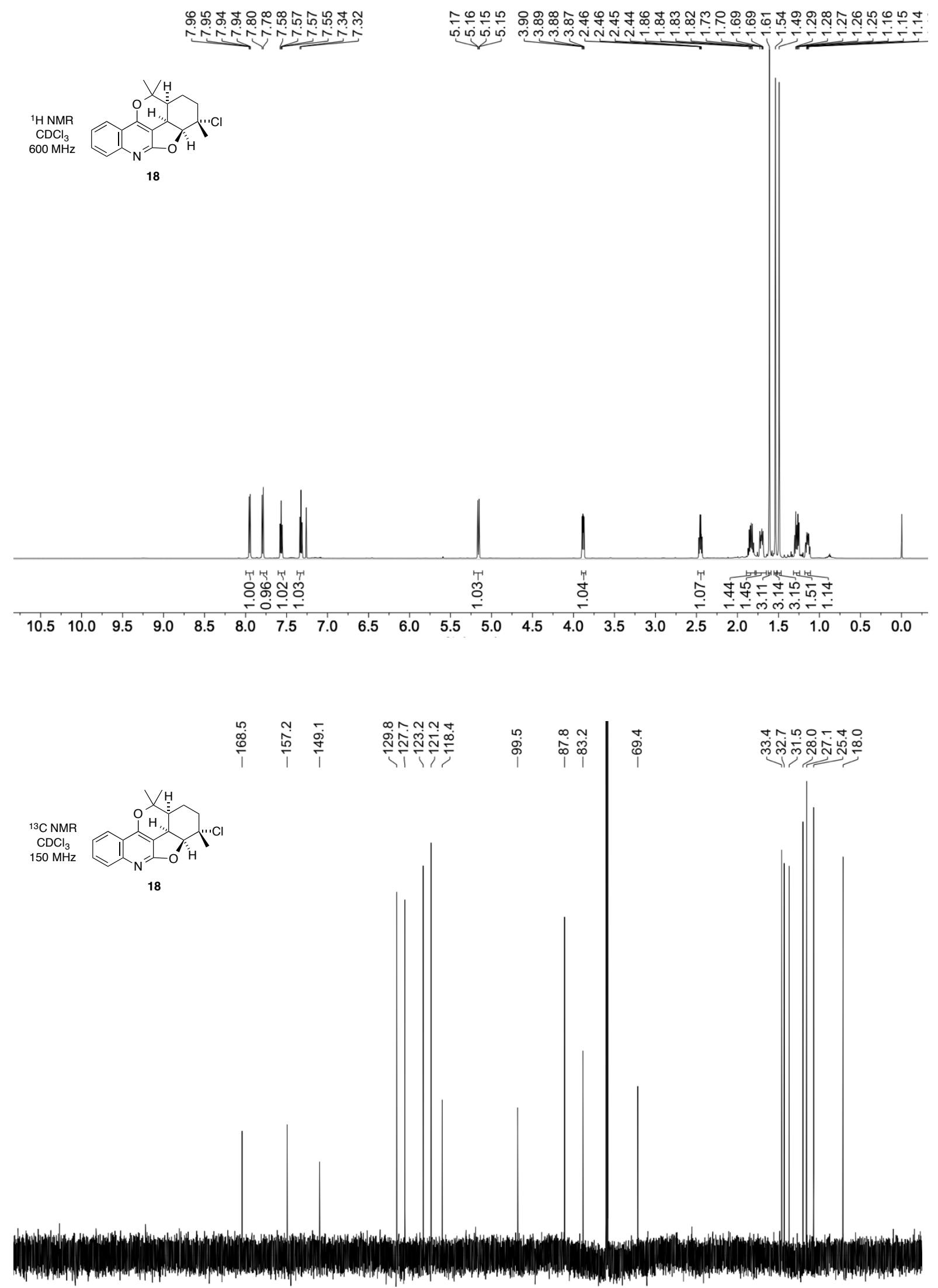

\begin{tabular}{lllllllllllllllllllllllll}
\hline 220 & 210 & 200 & 190 & 180 & 170 & 160 & 150 & 140 & 130 & 120 & 110 & 100 & 90 & 80 & 70 & 60 & 50 & 40 & 30 & 20 & 10 & 0
\end{tabular} 

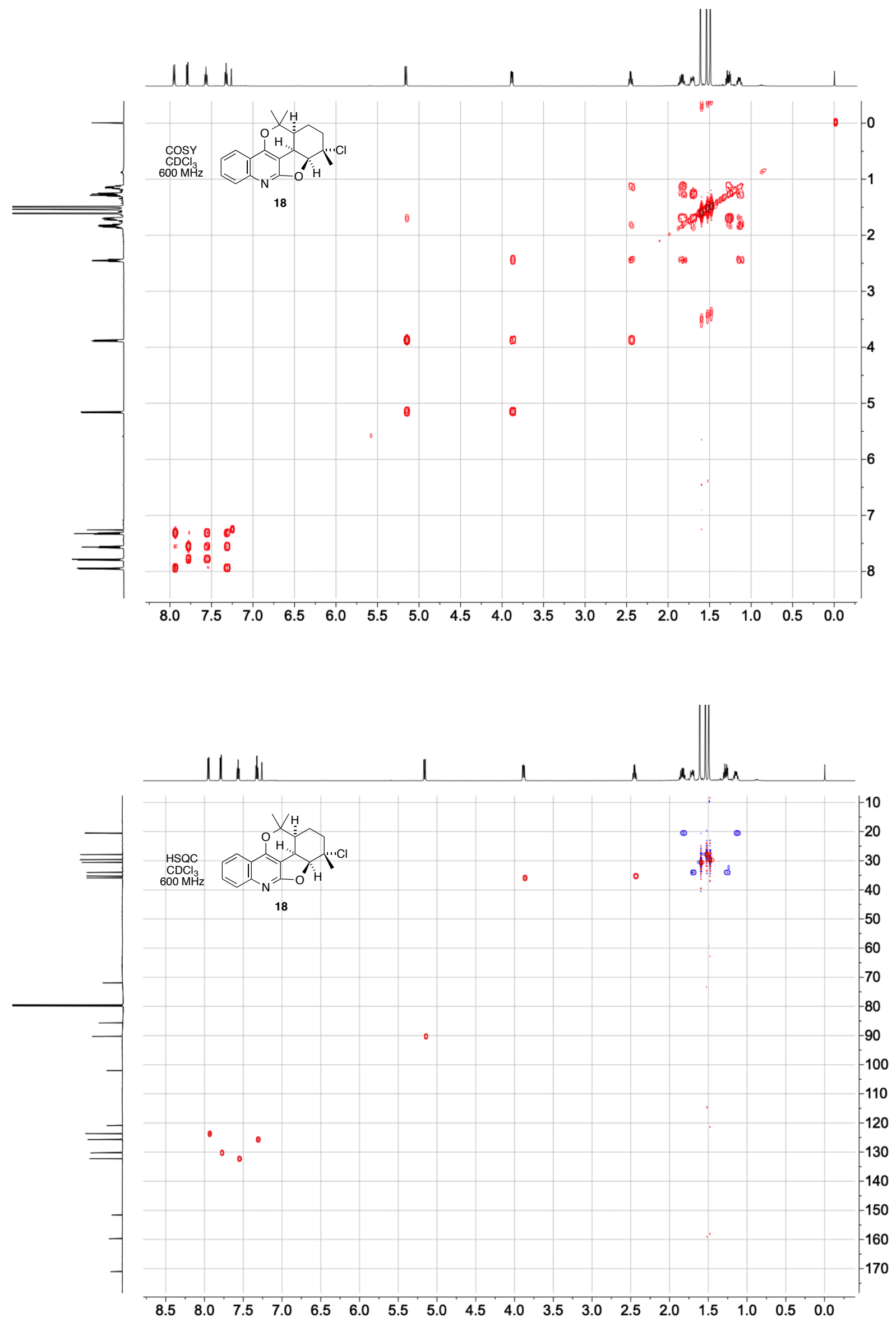

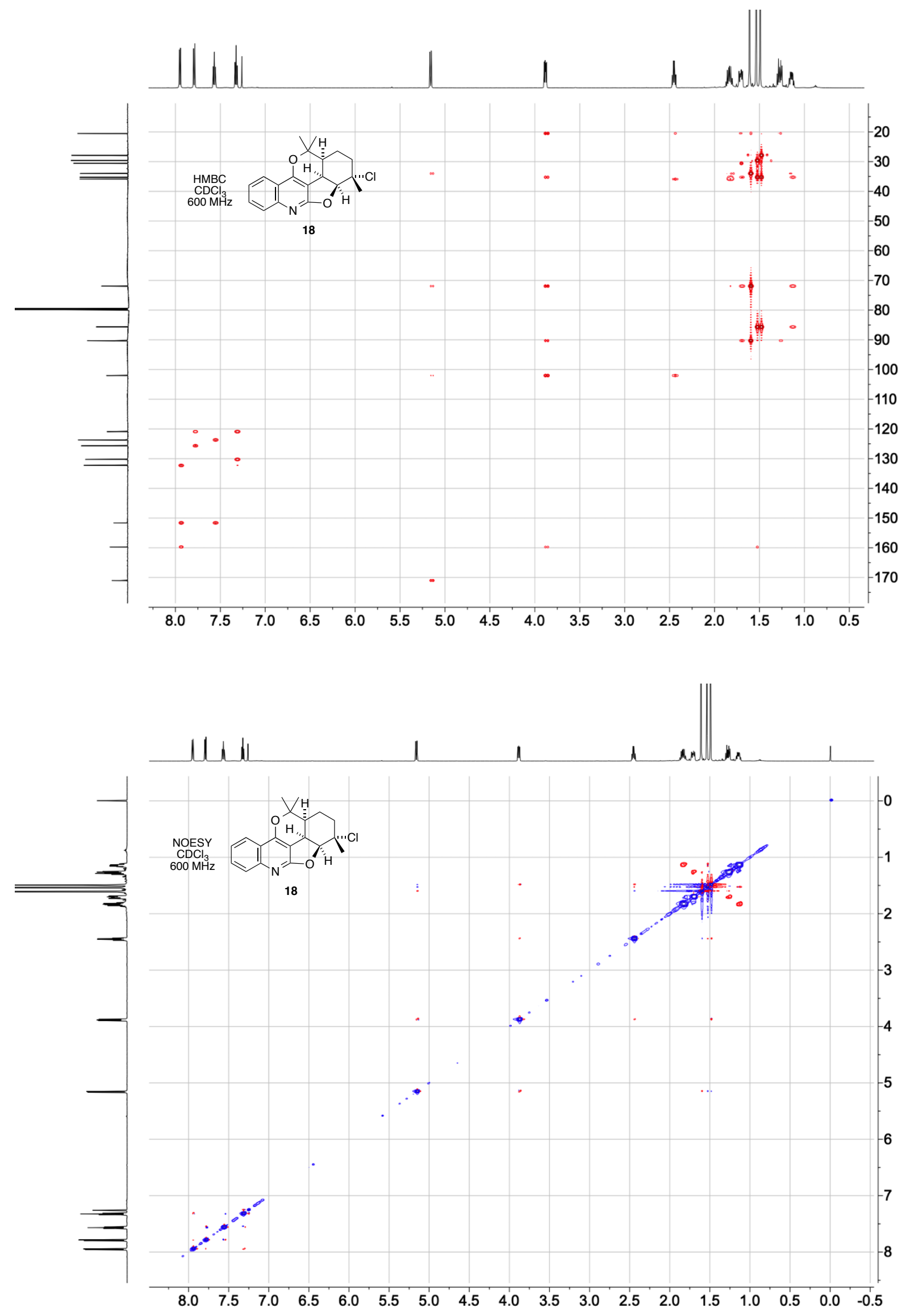


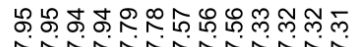

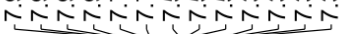

$1 \mathrm{H}$ NMR

$\mathrm{CDCl}_{3}$

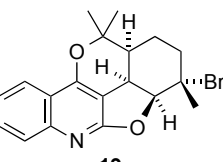

19
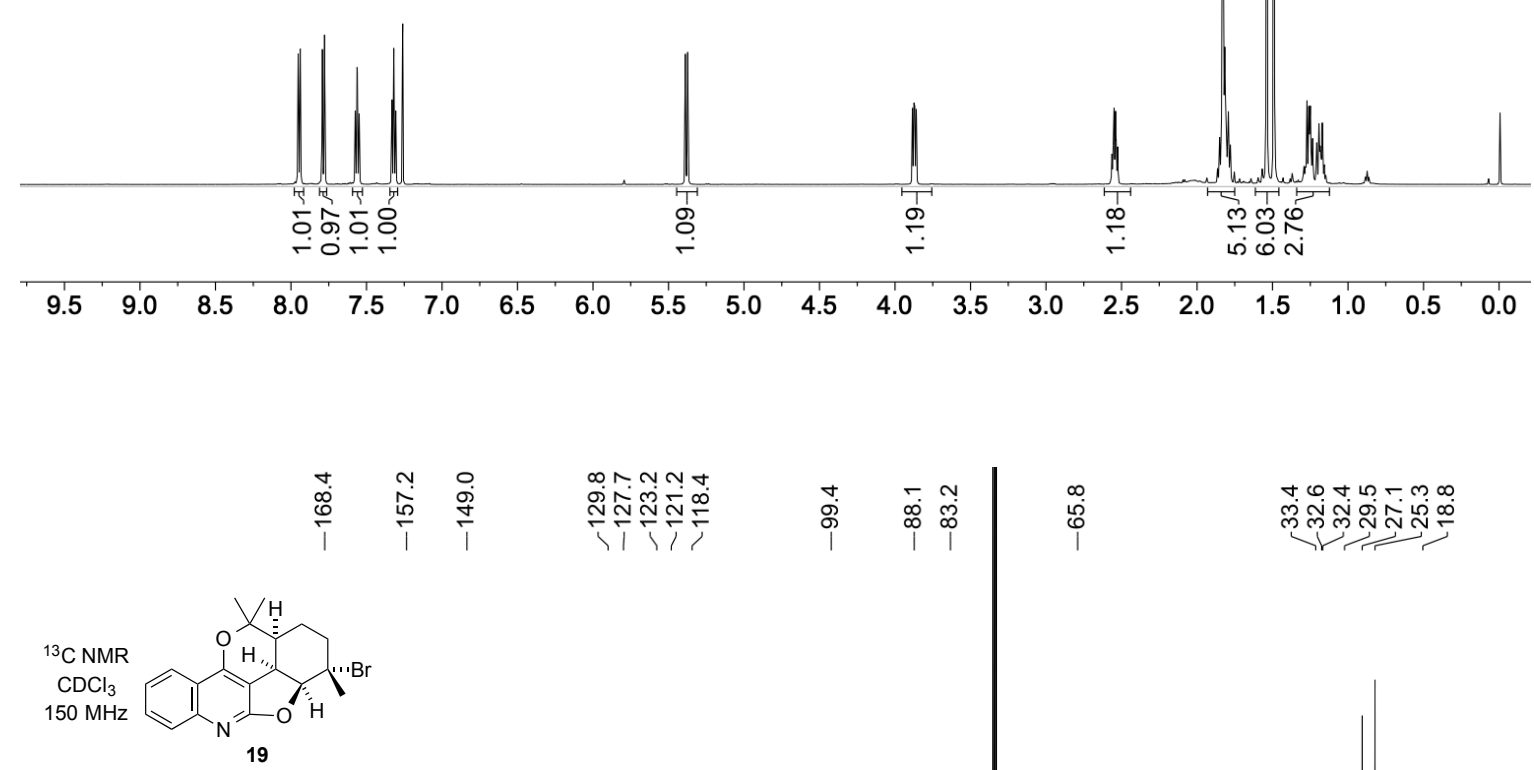

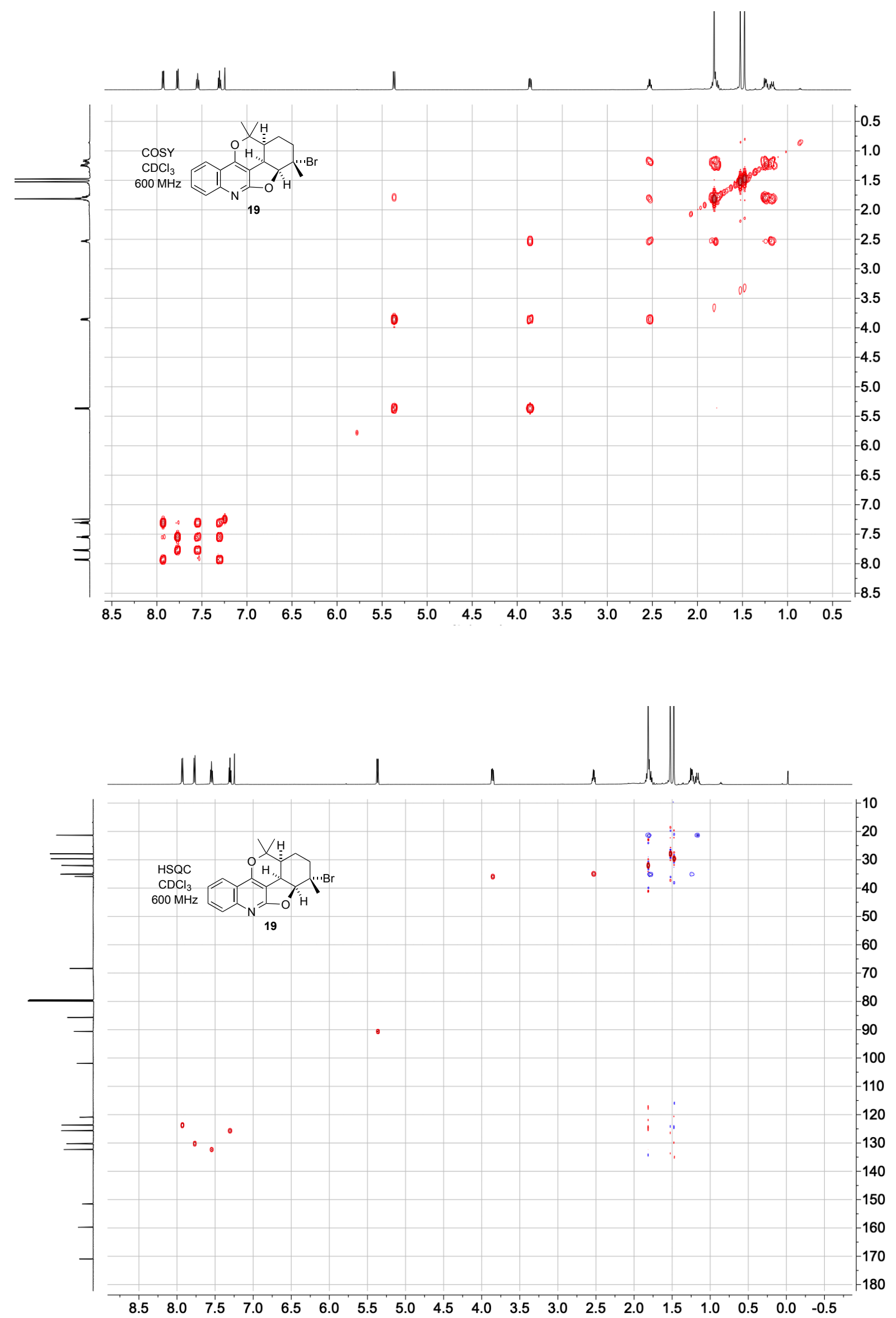

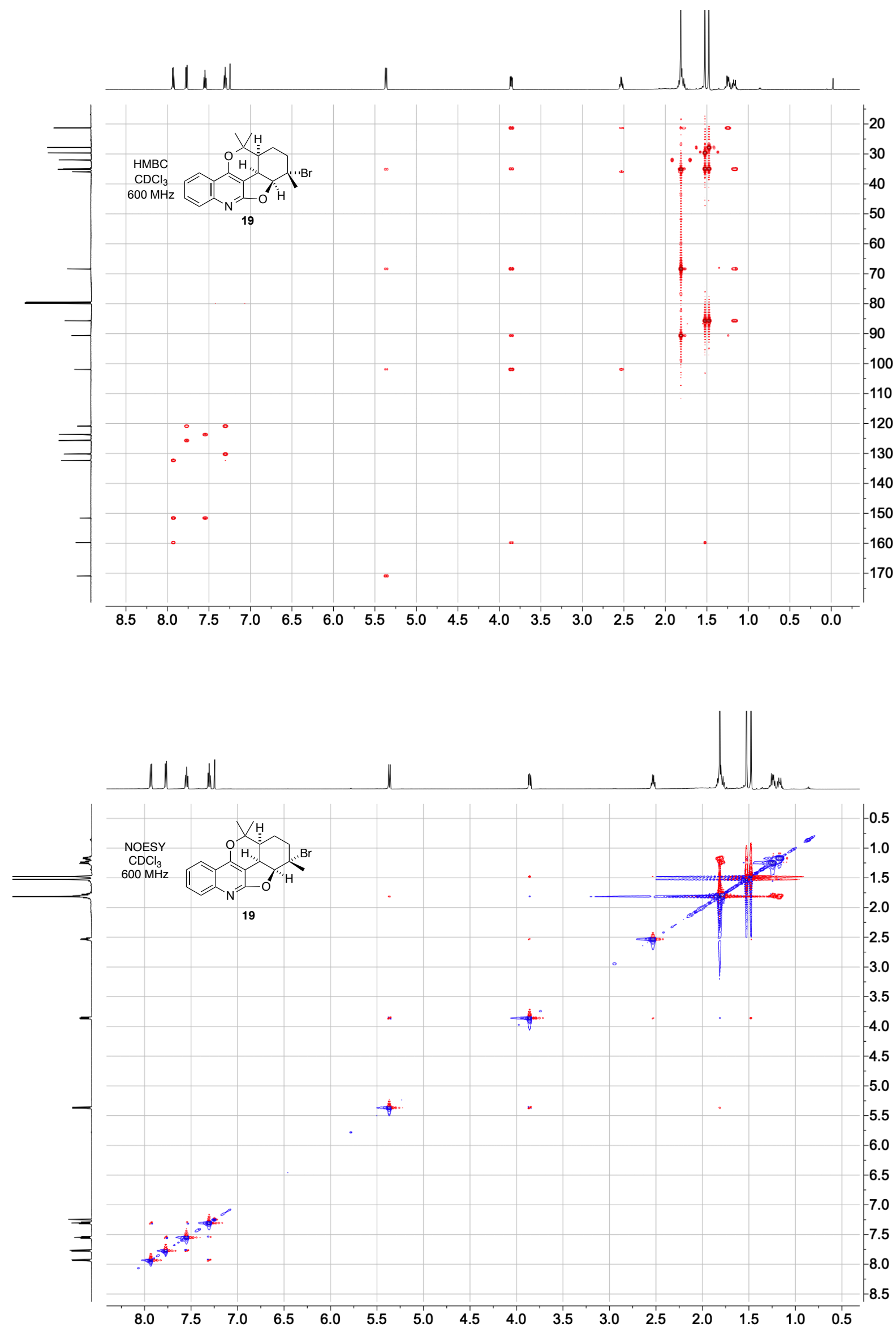


\section{Comparison of natural and synthetic NMR data}

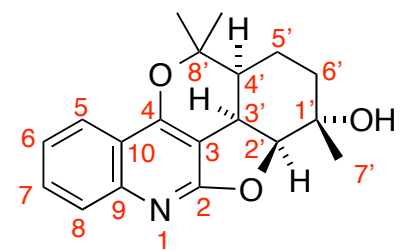

1: furoerioaustralasine

${ }^{1}$ H NMR

\begin{tabular}{|c|c|c|}
\hline Assignment & $\begin{array}{c}\text { Natural Sample, }{ }^{1} \mathrm{H} \text { NMR } \\
\left(400 \mathrm{MHz}, \mathrm{CDCl}_{\mathbf{3}}\right) \\
\text { Waterman et al. }^{2}\end{array}$ & $\begin{array}{c}\text { Synthetic Sample, }{ }^{1} \mathrm{H} \text { NMR } \\
\left.\text { (600 MHz, } \mathbf{C D C l}_{3}\right) \\
\text { This work }\end{array}$ \\
\hline 2 & -- & -- \\
\hline 3 & -- & -- \\
\hline 4 & -- & -- \\
\hline 5 & $7.95(\mathrm{dd}, J=8.0,2.0 \mathrm{~Hz})$ & $7.94(\mathrm{dd}, J=8.0,1.5 \mathrm{~Hz})$ \\
\hline 6 & $7.32(\mathrm{td}, J=8.0,2.0 \mathrm{~Hz})$ & $7.32(\mathrm{t}, J=7.5 \mathrm{~Hz})$ \\
\hline 7 & $7.56(\mathrm{td}, J=8.0,2.0 \mathrm{~Hz})$ & $7.56(\mathrm{t}, J=7.5 \mathrm{~Hz})$ \\
\hline 8 & $7.81(\mathrm{dd}, J=8.0,2.0 \mathrm{~Hz})$ & $7.80(\mathrm{~d}, J=8.4 \mathrm{~Hz})$ \\
\hline 9 & -- & -- \\
\hline 10 & -- & -- \\
\hline 1 ' & -- & -- \\
\hline $1 ' \mathrm{OH}$ & -- & -- \\
\hline 2 ' & $\begin{array}{c}3.84(\mathrm{~d}, J=9.2 \mathrm{~Hz}) \\
\text { We assume this value is a } \\
\text { typographical error. }\end{array}$ & $4.98(\mathrm{dd}, J=9.0,4.0 \mathrm{~Hz})$ \\
\hline $3^{\prime}$ & $3.84(\mathrm{dd}, J=9.2,6.3 \mathrm{~Hz})$ & $3.83(\mathrm{dd}, J=9.2,6.3 \mathrm{~Hz})$ \\
\hline 4 ' & $2.24(\mathrm{~m})$ & $2.24(\mathrm{dt}, J=10.6,7.0 \mathrm{~Hz})$ \\
\hline 5 & $\begin{array}{l}1.28(\mathrm{~m}) \\
1.78(\mathrm{~m})\end{array}$ & $\begin{array}{l}1.01(\mathrm{~m}) \\
1.78(\mathrm{~m}) \\
\end{array}$ \\
\hline 6 & $\begin{array}{l}1.28(\mathrm{~m}) \\
1.45(\mathrm{~m})\end{array}$ & $\begin{array}{l}1.33(\mathrm{~m}) \\
1.46(\mathrm{~m})\end{array}$ \\
\hline 7’Me & $1.08(\mathrm{~s})$ & $1.08(\mathrm{~s})$ \\
\hline 8 ' & -- & -- \\
\hline $8^{\prime} \alpha-\mathrm{Me}$ & $1.55(\mathrm{~s})$ & $1.55(\mathrm{~s})$ \\
\hline $8^{\prime} \beta-\mathrm{Me}$ & $1.55(\mathrm{~s})$ & $1.55(\mathrm{~s})$ \\
\hline
\end{tabular}




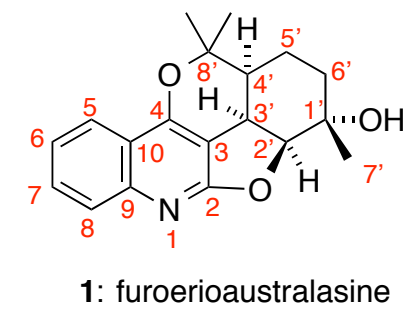

${ }^{13}$ C NMR

\begin{tabular}{|c|c|c|}
\hline Assignment & $\begin{array}{c}\text { Natural Sample, }{ }^{13} \mathrm{C} \mathrm{NMR} \\
\left(100 \mathrm{MHz}, \mathrm{CDCl}_{3}\right) \\
\text { Waterman et al. }^{2}\end{array}$ & $\begin{array}{c}\text { Synthetic Sample, }{ }^{13} \mathbf{C} \\
\text { NMR (150 MHz, CDCl }) \\
\text { This work }\end{array}$ \\
\hline 2 & 169.4 & 169.2 \\
\hline 3 & 100.3 & 100.2 \\
\hline 4 & 156.3 & 156.2 \\
\hline 5 & 121.2 & 121.1 \\
\hline 6 & 123.1 & 123.1 \\
\hline 7 & 129.6 & 129.6 \\
\hline 8 & 127.8 & 127.8 \\
\hline 9 & 149.2 & 149.2 \\
\hline 10 & 118.5 & 118.4 \\
\hline $1^{\prime}$ & 72.4 & 72.4 \\
\hline $2^{\prime}$ & 90.4 & 90.4 \\
\hline $3{ }^{\prime}$ & 33.7 & 33.7 \\
\hline $4 '$ & 35.7 & 35.6 \\
\hline 5 , & 18.6 & 18.5 \\
\hline 6 ' & 32.4 & 32.4 \\
\hline 7’'Me & 25.6 & 25.6 \\
\hline $8^{\prime}$ & 82.7 & 82.6 \\
\hline $8^{\prime} \alpha-\mathrm{Me}$ & 27.1 & 27.1 \\
\hline $8^{\prime} \beta-\mathrm{Me}$ & 25.7 & 25.7 \\
\hline
\end{tabular}




\section{Single crystal X-ray data}

A single crystal of 1 was mounted in paratone-N oil on a Mitigen crystal mount and X-ray diffraction data were collected at $150(2) \mathrm{K}$ on an Oxford X-calibur single crystal diffractometer using Mo $\mathrm{K} \alpha$ radiation. The data set was corrected for absorption using a multi-scan method, and the structures solved by direct methods using SHELXS-2008 and refined by full-matrix least squares on F2 by SHELXL-2015, ${ }^{3}$ interfaced through the programs X-Seed ${ }^{4}$ and Olex2 (Tables). ${ }^{5}$ Unless otherwise stated, non-hydrogen atoms were refined anisotropically and hydrogen atoms were included as invariants at geometrically estimated positions. Full data for the structure determination have been deposited with the Cambridge Crystallographic Data Centre as CCDC 1955472. Copies of this information may be obtained free of charge from The Director, CCDC, 12 Union Street, Cambridge CB2 1EZ, U.K. (fax, +44-1223-336-033; e-mail, deposit@,ccdc.cam.ac.uk). The table below provides the crystal data and structure refinement details for compound $\mathbf{1 .}$

3 (a) Sheldrick, G., SHELXS-2014 and SHELXL-2014. Program for X-ray Crystal Structure Determination, Göttingen University, Göttingen, Germany 2014. (b) Sheldrick, G. M. A short history of SHELX Acta Crystallographica Section A: Foundations of Crystallography 2008, 64, 112. (c) Sheldrick, G. M. Crystal structure refinement with SHELXL Acta Crystallographica Section C: Structural Chemistry 2015, 71, 3.

${ }^{4}$ Barbour, L. J. X-Seed - A Software Tool for Supramolecular Crystallography Journal of Supramolecular Chemistry 2001, 1, 189-191.

${ }^{5}$ Dolomanov, O. V.; Bourhis, L. J.; Gildea, R. J.; Howard, J. A.; Puschmann, H. OLEX2: a complete structure solution, refinement and analysis program Journal of Applied Crystallography 2009, 42, 339. 
Compound 1 crystallises in the monoclinic space group $P 2_{1} / c$ with two complete molecules in the asymmetric unit; these comprise a crystallographically independent hydrogen bonded dimer of 1 with one molecule of each isomer in the dimer, consistent with the compound being a racemic mixture. Both species have the trans relationship of the oxygen atoms resulting from $\mathrm{S}_{\mathrm{N}} 2$ ring-opening of epoxide. The figures below show a representation of one of the molecules in the asymmetric unit (thermal ellipsoids shown at the 50\% probability level), a view of a hydrogen bonded dimers with the intramolecular hydrogen bonds shown and finally a packing diagram of the structure viewed down the $b$-axis (slight offset).

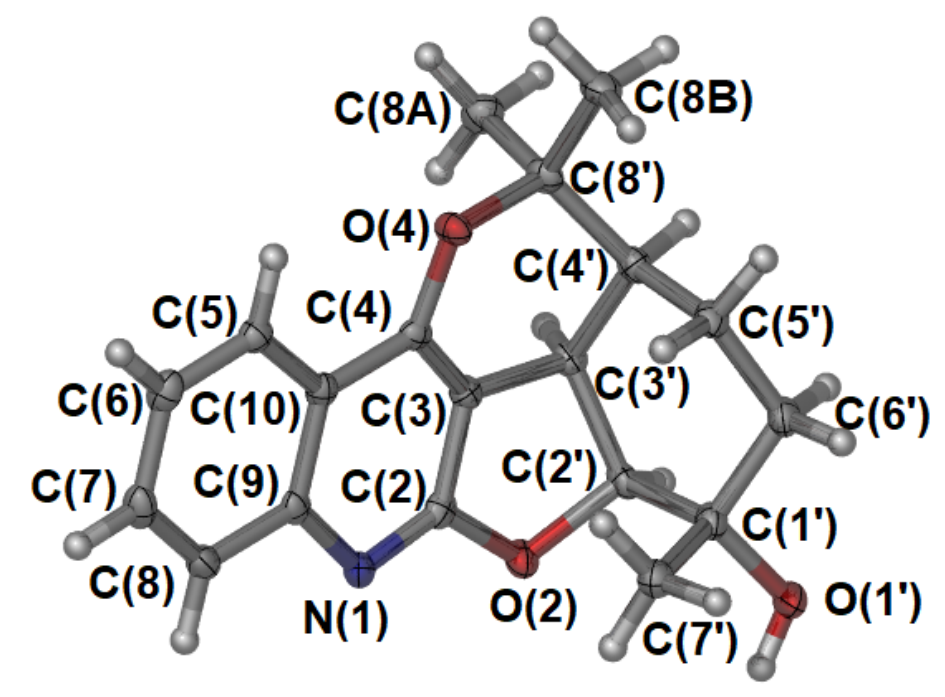

Figure S1. A perspective view of one selected molecule of the asymmetric unit of 1 with thermal ellipsoids shown at the 50\% probability level. Carbon - grey; hydrogen - white; oxygen - red; chlorine - yellow. 


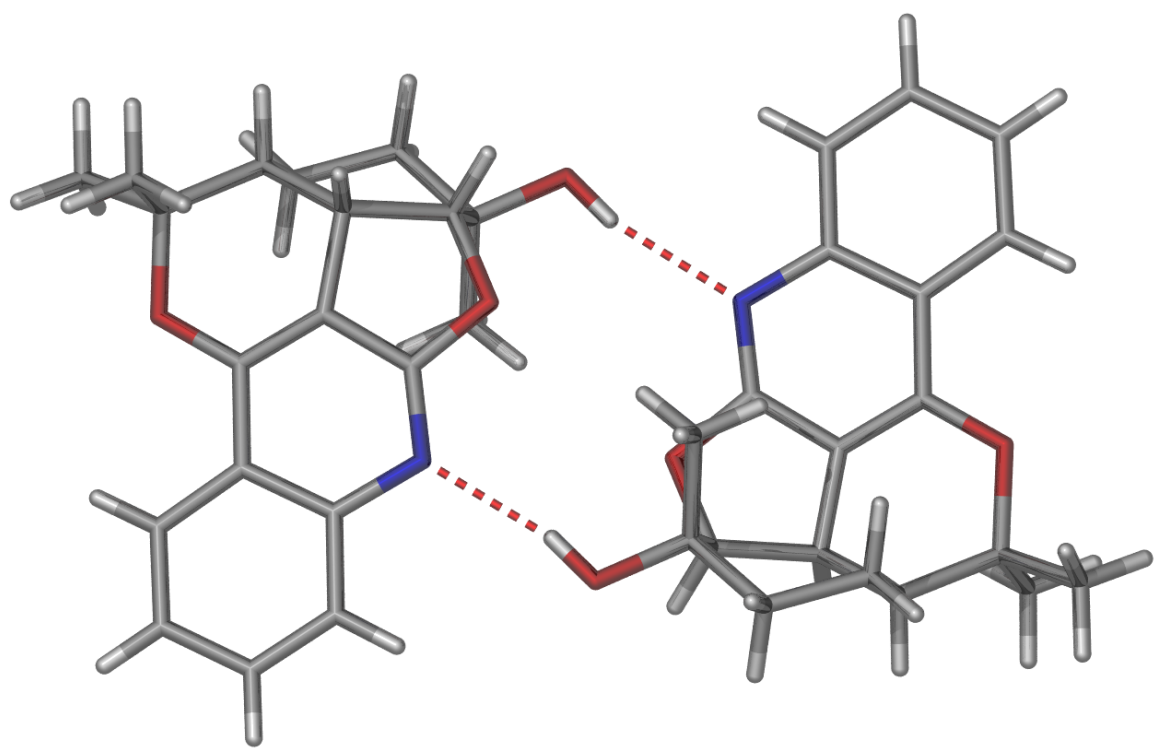

Figure S2. A perspective view of one of the hydrogen bonded dimers within the structure of 1. The hydrogen bonds are shown with the dashed lines.

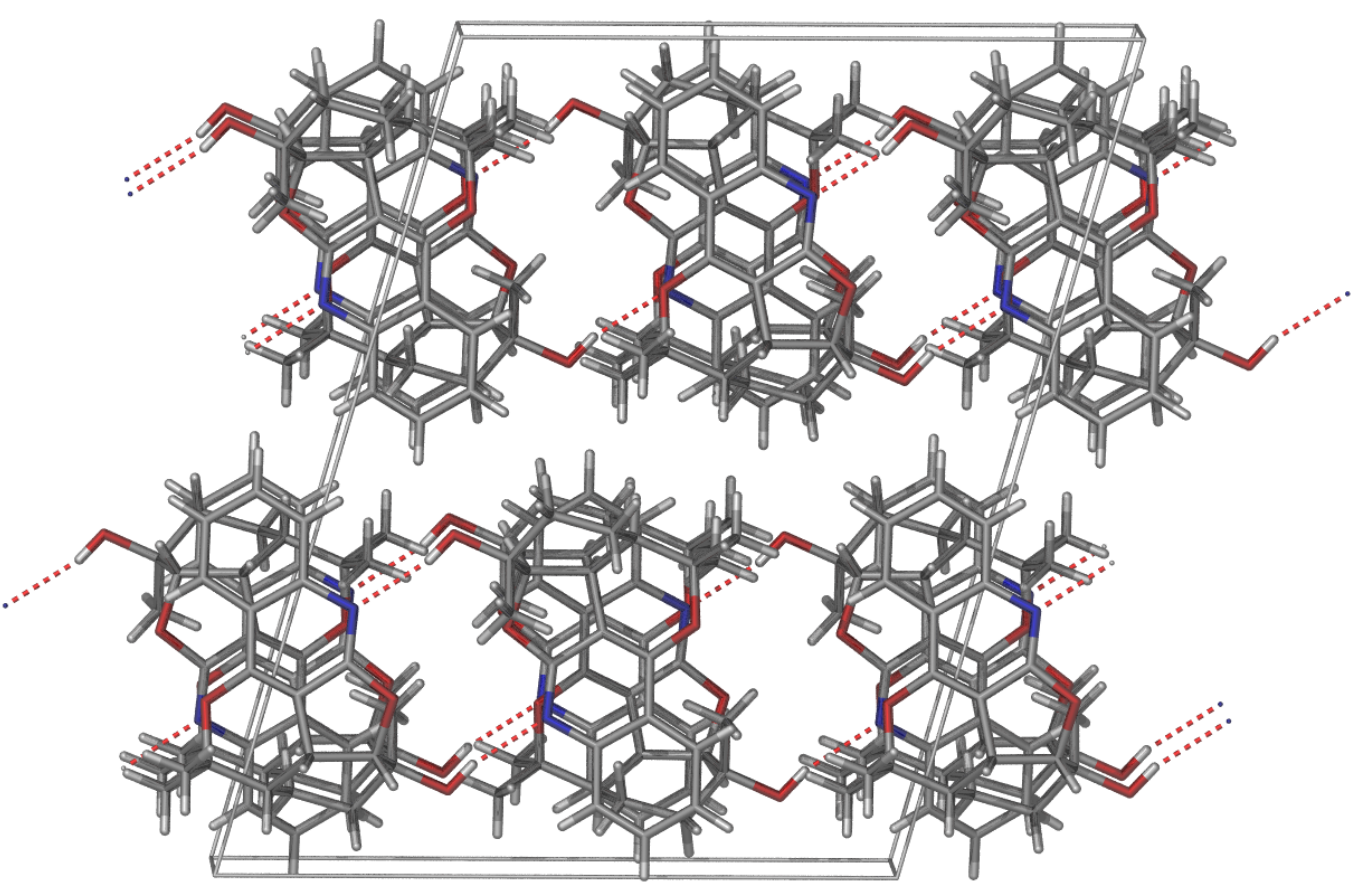

Figure S3. A view down the $b$-axis of the packing of compound 1. The unit cell is shown for reference. 
Table S1. Crystal data and structure refinement for $\mathbf{1}$.

\begin{tabular}{|c|c|}
\hline Identification code & Furo-MeOH \\
\hline CCDC number & 1955472 \\
\hline Empirical formula & $\mathrm{C}_{38} \mathrm{H}_{42} \mathrm{~N}_{2} \mathrm{O}_{6}$ \\
\hline Formula weight & 622.73 \\
\hline Temperature/K & $150(2)$ \\
\hline Crystal system & monoclinic \\
\hline Space group & $\mathrm{P} 21 / \mathrm{c}$ \\
\hline $\mathrm{a} / \AA \AA$ & $16.0238(8)$ \\
\hline $\mathrm{b} / \AA$ & $9.6558(4)$ \\
\hline $\mathrm{c} / \AA$ & $20.5379(8)$ \\
\hline$\alpha /^{\circ}$ & 90 \\
\hline$\beta /{ }^{\circ}$ & $106.176(4)$ \\
\hline$\gamma /{ }^{\circ}$ & 90 \\
\hline Volume $/ \AA^{3}$ & $3051.9(2)$ \\
\hline$Z$ & 4 \\
\hline$\rho_{\text {calc }} \mathrm{g} / \mathrm{cm}^{3}$ & 1.355 \\
\hline$\mu / \mathrm{mm}^{-1}$ & 0.091 \\
\hline $\mathrm{F}(000)$ & 1328.0 \\
\hline Crystal size $/ \mathrm{mm}^{3}$ & $0.72 \times 0.12 \times 0.05$ \\
\hline Radiation & $\operatorname{MoK} \alpha(\lambda=0.71073)$ \\
\hline $2 \Theta$ range for data collection $/{ }^{\circ}$ & 6.772 to 58.622 \\
\hline Index ranges & $-21 \leq \mathrm{h} \leq 21,-12 \leq \mathrm{k} \leq 11,-27 \leq 1 \leq 27$ \\
\hline Reflections collected & 33812 \\
\hline Independent reflections & $7466\left[\mathrm{R}_{\text {int }}=0.0695, \mathrm{R}_{\text {sigma }}=0.0940\right]$ \\
\hline Data/restraints/parameters & $7466 / 0 / 423$ \\
\hline Goodness-of-fit on $\mathrm{F}^{2}$ & 1.022 \\
\hline Final $R$ indexes $[\mathrm{I}>=2 \sigma(\mathrm{I})]$ & $\mathrm{R}_{1}=0.0594, \mathrm{wR}_{2}=0.1001$ \\
\hline Final $\mathrm{R}$ indexes [all data] & $\mathrm{R}_{1}=0.1197, \mathrm{wR}_{2}=0.1219$ \\
\hline Largest diff. peak/hole / e $\AA^{-3}$ & $0.27 /-0.29$ \\
\hline
\end{tabular}

